\title{
Accurate and efficient simulation of transport in multidimensional flow
}

\author{
B. Latorre*, P. Garcia-Navarro ${ }^{+}$, J. Murillo and J. Burguete \\ *e-mail: borja.latorre@unizar.es \\ +e-mail: pigar@unizar.es
}

\begin{abstract}
Fluid Mechanics. CPS. University of Zaragoza
50018 Zaragoza. Spain
\end{abstract}

\begin{abstract}
A new numerical method to obtain high order approximations of the solution of the linear advection equation in multidimensional problems is presented. The proposed conservative formulation is explicit and based on a single updating step. Piecewise polynomial spatial discretization using Legendre polynomials provides the required spatial accuracy. The updating scheme is built from the functional approximation of the exact solution of the advection equation and a direct evaluation of the resulting integrals. The numerical details for the schemes in one and two spatial dimensions are provided and validated using a set of numerical experiments. Test cases have been oriented to the convergence and the computational efficiency analysis of the schemes.
\end{abstract}

Keywords: high-order schemes, linear advection, multidimensional flow, CFA. 


\section{Introduction}

The study of mixing in fluid flows is a complex problem involving different phenomena that can be faced under different degrees of approximation. For many hydraulic and environmental applications it is widely assumed that the fate of a tracer concentration can be modeled by means of the differential tracer mass conservation equation. This contains information on the mechanism of advection by the average flow velocity as well as molecular diffusion and turbulence mixing. The latter are often formulated as general diffusion or dispersion terms in the equation [12]. Using the mass conservation equation for the fluid flow it can be simplified to get the most widely used non-conservative form known as the convection-dispersion equation.

It is possible to solve numerically the advection-diffusion equation by discretizing the complete equation or by solving separately the advection and the diffusion. In this work, letting aside the technique that could be applied to the diffusive part, we are concerned with an efficient and accurate treatment of the convective part.

A new approach is proposed here in order to provide high order approximations of the solution of the linear advection equation in multidimensional flow. The proposed conservative formulation is explicit and based on a single time step, does not require high order reconstruction techniques and provides information at sub-grid scale. The main characteristic of the method is that high order computation can be made efficient by the use of coarser grids, faster and more accurate than first order methods thanks to the sub-grid representation.

In general, finite difference methods are based on the differential form of the conservation law and a local approximation of the different derivatives. High order explicit schemes of this type provide accurate approximations of the spatial derivatives via a high order spatial reconstruction evaluating a large cell stencil. The time accuracy is achieved either using Taylor time series [7] or using a Runge-Kutta procedure [5] of at most 4th order [2]. These methods produce oscillations in the vicinity of sharp gradients and different limitation techniques are applied to the spatial reconstruction of the variables. One family of techniques are the second order TVD methods based on flux or slope limiters 
[10]. For higher orders the ENO and WENO techniques have been widely used [9].

Finite volume methods rely on an integral formulation of the conservation law. The evolution of the cell averaged value of the conserved variable is evaluated through an estimation of the fluxes at the cell edges. Godunov's method, for instance, evaluates the flux using the exact solution of the Riemann problem at the edge [4]. Several approximate Riemann solvers have been proposed in order to generate efficient schemes [11], [6]. High order finite volume methods use also high order spatial reconstructions at the grid cells in order to improve the evaluation of the numerical fluxes and Taylor time series or RungeKutta time integrations. The limiting procedure to avoid oscillations follows the same criteria than in finite differences. A well known second order finite volume method is the MUSCL-Hancock scheme [17]. Ben-Artzi [1] and Toro [14] proposed methods based on the solution of the generalized Riemann problem (piecewise polynomial), up to second order [1] and arbitrary high order (ADER) [14] [13] [15] in space and time. The ADER formulation offers the main advantage of requiring a single time step, however, it is associated to the necessity of following the steps of high order reconstruction, high order limitation, flux estimation and finally the storage of a single cell averaged value. This is a disadvantage common to all finite volume methods.

Dumbser [3] introduced the ADER methodology in the discontinuous Galerkin finite element framework. The variable is represented at every cell as a linear combination of basis functions leading to high order discretization avoiding the reconstruction step. Time accuracy is achieved in a single step thanks to the ADER formulation that transforms time derivatives into space derivatives. The finite element formulation projects the conservation law on every basis function in the time-space domain and evaluates the resulting integrals by means of quadrature formulae, allowing the resolution in both structured and unstructured grids.

This work presents a high order discretization technique analogous to the finite element methodology, based on Legendre polynomials. Among the different options available, these polynomials are the only leading to a conservative functional approximation. High order in space is achieved thanks to the polynomial representation within each cell avoiding the reconstruction step. High order in time is achieved in a single step by a conservative 
functional approximation (CFA technique) of the exact solution of the conservation law and a direct evaluation of the resulting integrals that does not require quadrature formulae. The resulting scheme is efficient in the sense that it requires a minimum number of mathematical operations. The scheme of order $N$ to solve the linear advection equation in $D$ dimensions requires $2 D N^{2 D}$ multiplications and additions per cell to calculate the evolution of the variable in a time step.

\section{CFA formulation for the linear advection equation in one dimension}

\subsection{Spatial discretization and sub-grid information}

Classical finite volume numerical schemes represent the state of a system by storing the average value of the variable at every grid cell and calculate the evolution of the system by means of an approximation of the updated average cell variable at a new time level. The numerical schemes considered in this work start from the basis that the information stored in a cell is a functional approximation, of a certain order of accuracy, of the spatial distribution of the variable within that cell. The numerical scheme is built to calculate the system evolution by providing an approximation of the new spatial distribution of the variable, at every cell, in the next time step.

The method will be described assuming, for the sake of clarity, a one-dimensional domain. The spatial representation of the variables at every grid cell is based on the mathematical concept of Hilbert space, an infinite-dimensional function space, defined over a spatial domain $x \in[a, b]$, with the following properties:

- The inner product of two square-integrable functions $f(x), g(x)$ is defined as:

$$
\langle f, g\rangle=\int_{a}^{b} f(x) g(x) d x
$$

- The norm of a function $f(x)$ is defined by: 


$$
\|f\|=\sqrt{\langle f, f\rangle}
$$

- Two functions $f(x), g(x)$ are said to be orthogonal whenever:

$$
\langle f, g\rangle=0
$$

- An orthogonal function basis is formed by an infinite set of functions $f_{n}(x)$ that:

$$
\left\langle f_{m}, f_{n}\right\rangle=\left\|f_{n}\right\|^{2} \delta_{m, n}
$$

- Any square-integrable function $g(x)$ can be expressed as linear combination of the elements of an orthogonal function basis $f_{n}(x)$ :

$$
g(x)=\sum_{n=1}^{\infty} g_{n} f_{n}(x)
$$

with the coefficients:

$$
g_{n}=\frac{\left\langle g, f_{n}\right\rangle}{\left\|f_{n}\right\|^{2}}
$$

Truncated series are used in practice and the number of orthogonal functions is related to the order of accuracy of the approximation.

The particular basis functions used in the present work are Legendre polynomials $P_{n}(x)$, defined in the spatial domain $x \in[-1,1]$. Their orthogonality is given by the property:

$$
\left\langle P_{m}, P_{n}\right\rangle=\int_{-1}^{1} P_{m}(x) P_{n}(x) d x=\frac{2}{2 n-1} \delta_{m, n}
$$

with the norm:

$$
\left\|P_{n}\right\|=\sqrt{\left\langle P_{n}, P_{n}\right\rangle}=\sqrt{\frac{2}{2 n-1}}
$$

Legendre polynomials can be constructed using the following recurrence rule: 


$$
n P_{n+1}(x)=(2 n-1) x P_{n}(x)-(n-1) P_{n-1}(x)
$$

Among them, the six first elements are:

$$
\begin{aligned}
P_{1}(x) & =1 & P_{2}(x) & =x \\
P_{3}(x) & =\frac{1}{2}\left(3 x^{2}-1\right) & P_{4}(x) & =\frac{1}{2}\left(5 x^{3}-3 x\right) \\
P_{5}(x) & =\frac{1}{8}\left(35 x^{4}-30 x^{2}+3\right) & P_{6}(x) & =\frac{1}{8}\left(63 x^{5}-70 x^{3}+15 x\right)
\end{aligned}
$$

One useful property of the Legendre polynomials in the context of the present work is that for $n>1$ they do not have net area:

$$
\int_{-1}^{1} P_{n}(x) d x=\int_{-1}^{1} P_{n}(x) P_{1}(x) d x=\left\langle P_{n}, P_{1}\right\rangle=2 \delta_{n, 1}
$$

An approximation of order of accuracy $N$ of a square-integrable function $g(x)$ in the interval $x \in[-1,1]$ can be obtained as linear combination of the $N$ first Legendre polynomials:

$$
g(x)=\sum_{n=1}^{N} g_{n} P_{n}(x)+O\left(x^{N}\right)
$$

with the coefficients:

$$
g_{n}=\frac{2 n-1}{2}\left\langle g, P_{n}\right\rangle
$$

The approximation is denoted by $\bar{g}(x)$ :

$$
\bar{g}(x)=\sum_{n=1}^{N} g_{n} P_{n}(x)
$$

It is important to note that the mentioned approximation $\bar{g}(x)$ is conservative. This property is fundamental to the conservative character of the numerical scheme developed and is exclusively associated to the use of the Legendre polynomials. To prove it, equation (13) is integrated using (12) and (10).

$$
\int_{-1}^{1} \bar{g}(x) d x=\sum_{n=1}^{N} g_{n} \int_{-1}^{1} P_{n}(x) d x=\sum_{n=1}^{N} g_{n}\left\langle P_{n}, P_{1}\right\rangle=2 g_{1}=\int_{-1}^{1} g(x) d x
$$


This property is useful to identify $g_{1}$ as the average value of function $g(x)$ in the interval $x \in[-1,1]$.

$$
g_{1}=\frac{1}{2} \int_{-1}^{1} g(x) d x=\frac{1}{2} \int_{-1}^{1} \bar{g}(x) d x
$$

Due to the restrictions on the domain where the Hilbert space is defined, it is necessary, to move from a global to a local coordinate system within each cell, that is adapted to the domain of orthogonality of the basis functions. For that purpose $x^{\prime}$ will be used to denote the global coordinate and $x$ to denote the local, cell adapted, coordinate.

Assuming for instance an spatial domain $x^{\prime} \in[0, L]$, with a grid of uniform cell size $\Delta x^{\prime}$ and an initial condition $q\left(x^{\prime}\right)$, the local coordinate transformation at the cell $i$ becomes

$$
x^{\prime}(x, i)=\frac{\Delta x^{\prime}}{2}(x+2 i+1)
$$

with the properties:

$$
x^{\prime}(-1, i)=i \Delta x^{\prime}, \quad x^{\prime}(1, i)=(i+1) \Delta x^{\prime}
$$

The spatial distribution of the variable is expressed in local coordinate

$$
q_{i}(x)=q\left(x^{\prime}(x, i)\right)=q\left(\frac{\Delta x^{\prime}}{2}(x+2 i+1)\right)
$$

and is associated to the following approximation of order of accuracy $N$ :

$$
\bar{q}_{i}(x)=\sum_{n=1}^{N}{ }^{n} q_{i} P_{n}(x) \simeq q_{i}(x)
$$

with the coefficients:

$$
{ }^{n} q_{i}=\frac{2 n-1}{2} \int_{-1}^{1} q_{i}(x) P_{n}(x) d x
$$

The coefficients in (20) carry the information of the initial condition approximation. The particular value ${ }^{1} q_{i}$ represents the average value of $\bar{q}_{i}(x)$ in the cell $i$.

To summarize, for the scheme of order of accuracy $N$, the state of the system at time $t$ will be represented by means of the storage of $N$ numbers at every grid cell ${ }^{n} q_{i}^{t}$ 
representing the spatial distribution of the variable as linear combination of the Legendre polynomials:

$$
q_{i}(x, t)=\sum_{n=1}^{N}{ }^{n} q_{i}^{t} P_{n}(x)
$$

To illustrate this, consider the approximation of the initial condition

$$
q\left(x^{\prime}, 0\right)=e^{\left(-\left(x^{\prime}-1\right)^{2} / 0.05\right)}
$$

in the domain $x^{\prime} \in[0,2]$, assuming a discretization in $M$ grid cells. The resulting first order approximation using $M=30$ cells is compared with the third order representation using $M=10$ cells in Figure 1. The two representations are of different quality although they are based on the same amount of stored information (30 numbers), being the third order approximation preferable. This is analogous to the theory supporting the most efficient image and video compression algorithms.

\subsection{Time evolution}

The form to build a numerical scheme able to solve the time evolution of a given spatial distribution based on the Legendre polynomial representation is next presented. The linear advection equation is chosen in order to state the basic features of the method that, afterwards, can be extended to more complex equations and ambiguous applications.

The linear advection equation in one dimension for a function $q\left(x^{\prime}, t\right)$ is:

$$
\partial_{t} q\left(x^{\prime}, t\right)+\lambda \partial_{x} q\left(x^{\prime}, t\right)=0
$$

Instead of seeking an approximation of the individual terms in the equation, the existing exact solution is used as the basis of the advective method. The time evolution step is analogous to the semi-Lagrangian or Eulerian-Lagrangian method [18]. The exact solution can be expressed in global coordinates:

$$
q\left(x^{\prime}, t+\Delta t\right)=q\left(x^{\prime}-\lambda \Delta t, t\right)
$$

and transformed in local coordinate: 


$$
q(x, t+\Delta t)= \begin{cases}q(x-2 c, t) & \text { if } \lambda \geq 0 \\ q(x+2 c, t) & \text { if } \lambda \leq 0\end{cases}
$$

where $c$ is the $C F L$ number:

$$
c=\frac{|\lambda| \Delta t}{\Delta x^{\prime}}
$$

Hence, starting from the known initial conditions, already expressed in local coordinates (21), we are interested in an expression for the solution at time $t=t+\Delta t$.

To achieve that, the pure transport formulated by (25) of the initial condition (21) is performed leading to the exact solution in cell $i$ as follows for $\lambda>0$ :

$$
\widetilde{q}_{i}(x, t+\Delta t)= \begin{cases}q_{i-1}(x+2-2 c, t) & \text { if }-1<x<2 c-1 \\ q_{i}(x-2 c, t) & \text { if } 2 c-1<x<1\end{cases}
$$

and as follows for $\lambda<0$

$$
\widetilde{q}_{i}(x, t+\Delta t)= \begin{cases}q_{i}(x+2 c, t) & \text { if }-1<x<1-2 c \\ q_{i+1}(x-2+2 c, t) & \text { if } 1-2 c<x<1\end{cases}
$$

Finally a conservative functional approximation of $(27),(28)$ is performed in order to reach the updated set of coefficients in the grid cell:

$$
q_{i}(x, t+\Delta t)=\sum_{n=1}^{N}{ }^{n} q_{i}^{t+\Delta t} P_{n}(x)
$$

with the coefficients:

$$
{ }^{n} q_{i}^{t+\Delta t}=\frac{2 n-1}{2} \int_{-1}^{1} \widetilde{q}_{i}(x, t+\Delta t) P_{n}(x) d x
$$

The three main steps in the method are sketched in Figure 2. Starting from the initial condition (21), the transported exact solution at the new time step is obtained (27), (28) and finally approximated (29). 


\subsection{Updating scheme in one dimension}

The exact calculation of the integrals present in the definition of the coefficients (30), as detailed in Appendix A, leads to the following updating numerical scheme of order accuracy $N$ :

$$
{ }^{n} q_{i}^{t+\Delta t}= \begin{cases}\sum_{j=1}^{N}{ }^{j} q_{i-1}^{t} L_{n, j}(c)+\sum_{j=1}^{N}{ }^{j} q_{i}^{t} R_{n, j}(c) & \text { if } \lambda \geq 0 \\ \sum_{j=1}^{N}{ }^{j} q_{i}^{t} L_{n, j}(1-c)+\sum_{j=1}^{N}{ }^{j} q_{i+1}^{t} R_{n, j}(1-c) & \text { if } \lambda \leq 0\end{cases}
$$

Vector notation can be used for convenience:

$$
\vec{q}_{i}^{t+\Delta t}= \begin{cases}L(c) \vec{q}_{i-1}^{t}+R(c) \vec{q}_{i}^{t} & \text { if } \lambda \geq 0 \\ L(1-c) \vec{q}_{i}^{t}+R(1-c) \vec{q}_{i+1}^{t} & \text { if } \lambda \leq 0\end{cases}
$$

where:

$$
\vec{q}_{i}^{t}=\left(\begin{array}{c}
{ }^{1} q_{i}^{t} \\
{ }^{2} q_{i}^{t} \\
\cdots \\
{ }^{N} q_{i}^{t}
\end{array}\right)
$$

In (31) $c$ is the $C F L$ number (26) used to evaluate the left matrix $L$ :

$$
L(c)=T(c, 1-c)
$$

and the right matrix $R$ :

$$
R(c)=T(1-c,-c)
$$

Both $L$ and $R$ matrix are written in terms of a translation matrix $T$ :

$$
T_{i, j}(a, b)=a \frac{2 i-1}{2} \sum_{n=1}^{\min (i, j)} \frac{2}{2 n-1} A_{i, n}(a,-b) A_{j, n}(a, b)
$$

where $A$ is a matrix with the property: 


$$
P_{i}(a x+b)=\sum_{n=1}^{N} A_{i, n}(a, b) P_{n}(x)
$$

Note that $A_{i, j}=0$ if $j>i$ thus reducing the sum elements in (36) to $\min (i, j)$.

To illustrate the procedure, the expression of the auxiliary matrix $A$ up to third order of accuracy is next provided:

$$
A(a, b)=\left(\begin{array}{ccc}
1 & 0 & 0 \\
b & a & 0 \\
d & 3 a b & a^{2}
\end{array}\right), \quad d=\frac{a^{2}+3 b^{2}-1}{2}
$$

and the corresponding $T$ matrix:

$$
T(a, b)=a\left(\begin{array}{ccc}
1 & b & d \\
-3 b & a^{2}-3 b^{2} & 3\left(a^{2} b-b d\right) \\
5 d & 5\left(b d-a^{2} b\right) & a^{4}-15 a^{2} b^{2}+5 d^{2}
\end{array}\right), \quad d=\frac{a^{2}+3 b^{2}-1}{2}
$$

The numerical scheme (31) is conditionally stable provided that $c \leq 1$ for all orders of accuracy. This is dictated by the advection rule assumed (27) that only holds for $c \leq 1$. The scheme is exact when $c=1$ in the sense that it transports exactly the polynomial distribution within every grid cell.

In the particular case of using $N=1$ and assuming for simplicity $\lambda>0$, the scheme (31) reduces to the first order upwind scheme:

$$
{ }^{1} q_{i}^{t+\Delta t}={ }^{1} q_{i-1}^{t} c+{ }^{1} q_{i}^{t}(1-c)
$$

involving a single coefficient per grid cell. When developing the second order case, $N=2$ and assuming again $\lambda>0$, the scheme becomes:

$$
\begin{aligned}
\left(\begin{array}{c}
{ }^{1} q_{i}^{t+\Delta t} \\
{ }^{2} q_{i}^{t+\Delta t}
\end{array}\right)= & c\left(\begin{array}{cc}
1 & 1-c \\
-3(1-c) & c^{2}-3(1-c)^{2}
\end{array}\right)\left(\begin{array}{c}
{ }^{1} q_{i-1}^{t} \\
{ }^{2} q_{i-1}^{t}
\end{array}\right) \\
& +(1-c)\left(\begin{array}{cc}
1 & -c \\
3 c & (1-c)^{2}-3 c^{2}
\end{array}\right)\left(\begin{array}{c}
{ }^{1} q_{i}^{t} \\
{ }^{2} q_{i}^{t}
\end{array}\right)
\end{aligned}
$$


involving now two coefficients per cell that are necessary to represent the solution at the subgrid scale.

It is worth noting that in a simulation with fixed $\Delta t$ and $\Delta x$, matrices $L$ and $R$ are constant. The scheme of order of accuracy $N$ requires in this case $2 N^{2}$ multiplications and additions per cell and time step. Taking in to account that the maximum time step allowed is independent of the order of accuracy, the total computational cost grows with the order of accuracy at a rate $N^{2}$.

\section{Numerical tests in one dimension}

\subsection{Order of accuracy and efficiency}

\subsubsection{Test 1}

A Gaussian initial distribution is used as first test case to quantify the actual order of accuracy of the different approximations.

$$
q\left(x^{\prime}, 0\right)=e^{-\left(x^{\prime}-1\right)^{2} / 0.05}
$$

A domain $x^{\prime} \in[0,2]$ is considered and periodical boundary conditions assumed. The advection velocity is chosen $\lambda=1$ and a simulation time of $t=20$ is performed so that the Gaussian distribution crosses 10 times the computational domain. The exact solution is the initial distribution at the same location. Using $c=0.95$, simulations with different cell size $\Delta x$ have been performed and their error has been evaluated using the $L_{1}$ norm as follows:

$$
L_{1}=\frac{1}{2 M} \sum_{i=1}^{M} \int_{-1}^{1}\left|q_{i}(x)-q_{\text {Exact }}(x)\right| d x
$$

where $M$ represent the cell number and the integrals in (43) are numerically computed.

The $L_{1}$ error norm corresponding to numerical schemes of orders of accuracy 2, 3, 4 and 5 versus the grid spacing is represented in logarithmic scale in Figure 3. In all the cases, the results have been fit to a straight line and the slope found is indicated in the figure. 


\subsubsection{Test 2}

The same initial distribution (42) and the same domain $x^{\prime} \in[0,2]$ are considered with the focus on the computational efficiency of the different schemes. We are interested in comparing the computational time required by the different schemes to achieve a target computational accuracy. The error of the schemes is quantified using the $L_{1}$ norm (43). Figure 4 displays the shape of the numerical solutions corresponding to values of $\log \left(L_{1}\right)=$ -1 and $\log \left(L_{1}\right)=-2$ compared to the exact solution in order to state the acceptable size of this error in practical applications.

Two sets of computations, corresponding to a simulation time of $t=20$ and $t=200$, have been made and are plotted in Figures 5 a) and 5 b) respectively. These figures are a representation of the $L_{1}$ errors produced by the CFA schemes of orders of accuracy 1, 2, 3, 4 and 5 as a function of the computational time used, in logarithmic scale. In general, given a desired maximum error there is a scheme of a certain order of accuracy able to provide the solution at the lowest cost. Also, the figures show that if, when starting by a first order approach the numerical error is excessive, refining the grid is never the best option to reduce the error. This tendency continues and indicates that, for longer simulations (either in larger domains) higher order approximations gain in relative efficiency.

\subsection{Monotonicity}

Other initial distributions including discontinuities in the function and in its derivative are now considered in order to analyze the monotonicity of the solutions provided by the different approximations. A computational domain $x^{\prime} \in[0,2]$ with periodical boundaries will be assumed.

\subsubsection{Test 3}

A first simulation with a uniform speed $\lambda=1$, a simulation time of $t=200$, and $c=0.5$ is performed as Test 3 assuming three different initial conditions: 


$$
\begin{aligned}
\text { Test 3.a }) & q\left(x^{\prime}, 0\right)=\ln (2) \frac{\left(x^{\prime}-1\right)^{2}}{0.04} \\
\text { Test 3.b }) & q\left(x^{\prime}, 0\right)= \begin{cases}1 & \text { if } 0.7<x^{\prime}<1.3 \\
0 & \text { otherwise }\end{cases} \\
\text { Test 3.c) } & q\left(x^{\prime}, 0\right)= \begin{cases}\sqrt{1-\left(x^{\prime}-1\right)^{2} / 0.09} & \text { if } 0.7<x^{\prime}<1.3 \\
0 & \text { otherwise }\end{cases}
\end{aligned}
$$

Figures 6 a), 6 b) and 6 c) show the results obtained with the CFA second order method, using $M=100$ cells, and the second order schemes of Lax-Wendroff and Upwind [10] as representative of the standard second order finite volume techniques, using $M=$ 200 cells. A different number of cells has been used in the CFA method in order to enforce the same amount of stored information in the three methods (200 numbers). The plot in Figures 6 a) displays cell average values (15) of the linear numerical solution.

All the schemes of order above 1 show an oscillatory behavior near steep gradients. To avoid them, the schemes need some kind of high order limitation or filtering [8]. In the present work a slope limiting technique has been used according to:

$$
{ }^{2} q_{i}^{\text {lim }}= \begin{cases}\frac{1}{2}\left(\max \left({ }^{1} q_{i+1},{ }^{1} q_{i}+{ }^{2} q_{i}\right)-\min \left({ }^{1} q_{i-1},{ }^{1} q_{i}-{ }^{2} q_{i}\right)\right) & \text { if }{ }^{1} q_{i-1}>{ }^{1} q_{i+1} \\ \frac{1}{2}\left(\min \left({ }^{1} q_{i+1},{ }^{1} q_{i}+{ }^{2} q_{i}\right)-\max \left({ }^{1} q_{i-1},{ }^{1} q_{i}-{ }^{2} q_{i}\right)\right) & \text { if }{ }^{1} q_{i-1}<{ }^{1} q_{i+1}\end{cases}
$$

where it must be taken into account that ${ }^{1} q_{i}$ represents the cell average value, ${ }^{2} q_{i}$ is the slope of the function in the local coordinates and ${ }^{1} q_{i} \pm{ }^{2} q_{i}$ provides the cell edge values. This limitation is imposed to the slopes of the updated solution at every time step modifying it when necessary. The basic method as described in the previous section is then used again in the next time step. This slope limitation does not alter the conservative character of the method. 
Figure $6 \mathrm{~d}$ ) shows the results obtained with the CFA second order slope limited method using $M=100$ cells.

\subsubsection{Test 4}

A compound initial shape made of a Gaussian, a square, a triangle and a semicircle is used as in [16].

$$
q\left(x^{\prime}, 0\right)= \begin{cases}\ln (2) \frac{\left(x^{\prime}-0.4\right)^{2}}{9 \cdot 10^{-4}} & \text { if } 0.3<x^{\prime}<0.5 \\ 1 & \text { if } 0.7<x^{\prime}<0.9 \\ 1-\sqrt{100\left(x^{\prime}-1.2\right)^{2}} & \text { if } 1.1<x^{\prime}<1.3 \\ \sqrt{1-100\left(x^{\prime}-1.6\right)^{2}} & \text { if } 1.5<x^{\prime}<1.7 \\ 0 & \text { otherwise }\end{cases}
$$

This distribution is transported at a uniform speed $\lambda=1$ during $t=20$. The simulation is performed with a fixed time step corresponding to $c=0.95$.

Figure 7 shows the longitudinal profiles of the cell averages obtained using $M=200$ cells with the CFA schemes of orders of accuracy 1, 2, 3, 4, 5 and 10 respectively and their comparison with the exact solution. From the available subgrid information, every dot in the graphical representation corresponds to the cell average value of the numerical solution (15). The same, as computed in a $M=50$ cell grid is displayed in Figure 9. The most noticeable feature of the numerical solutions is the Gibbs phenomenon near the discontinuities that increases in frequency as the order of accuracy grows, maintaining the size of the amplitude. This can be better observed in a zoom view of the numerical solution near the squared wave, as displayed in Figures 8 and 10. Finally, to stress the fact that the method provides subgrid information, Figure 11 shows the complete longitudinal profile of the solution calculated with the CFA scheme of order of accuracy 20. It is worth noting that the space interval is $\Delta x^{\prime}=0.2$, hence containing only 10 cells. 


\section{CFA formulation for the linear advection equation in two dimensions}

\subsection{Spatial discretization}

The spatial representation of the variable at every grid cell in two dimensions is based on a Hilbert space defined over a spatial domain $x \in[-1,1], y \in[-1,1]$ and the bases of Legendre polynomials. Given an initial distribution $q(x, y)$ defined in that domain, an approximation of order of accuracy $N$ can be obtained as:

$$
\bar{q}(x, y)=\sum_{m=1}^{N} \sum_{n=1}^{N} q_{m, n} P_{m}(x) P_{n}(y) \simeq q(x, y)
$$

where the coefficients:

$$
q_{m, n}=\frac{(2 m-1)(2 n-1)}{4} \int_{-1}^{1} \int_{-1}^{1} q(x, y) P_{m}(x) P_{n}(y) d x d y
$$

As in the one dimensional case is necessary to move from the global coordinate $\left(x^{\prime}, y^{\prime}\right)$ to local coordinate $(x, y)$ within each cell, that is adapted to the domain of orthogonality of the basis functions. Considering, for instance, a square domain $x^{\prime} \in[0, L], y^{\prime} \in[0, L]$ and a discretization $\Delta x^{\prime}, \Delta y^{\prime}$. The local coordinate transformation at cell $(i, j)$ is:

$$
\begin{aligned}
& x^{\prime}(x, i)=\frac{\Delta x^{\prime}}{2}(x+2 i+1) \\
& y^{\prime}(y, j)=\frac{\Delta y^{\prime}}{2}(y+2 j+1)
\end{aligned}
$$

and the following notation is implicit:

$$
q_{i, j}(x, y)=q\left(x^{\prime}(x, i), y^{\prime}(y, j)\right)
$$

For the scheme of order of accuracy $N$, the state of the system at time $t$ will be represented by means of the storage of $N^{2}$ numbers at every grid cell ${ }^{m, n} q_{i, j}^{t}$ representing the spatial distribution of the variable as linear combination of the Legendre polynomials: 


$$
q_{i, j}(x, y, t)=\sum_{m=1}^{N} \sum_{n=1}^{N}{ }^{m, n} q_{i, j}^{t} P_{m}(x) P_{n}(y)
$$

\subsection{Time evolution}

The extension of the linear advection equation to a two-dimensional transport is:

$$
\partial_{t} q\left(x^{\prime}, y^{\prime}, t\right)+\lambda_{x} \partial_{x} q\left(x^{\prime}, y^{\prime}, t\right)+\lambda_{y} \partial_{y} q\left(x^{\prime}, y^{\prime}, t\right)=0
$$

Where, now $\lambda=\left(\lambda_{x}, \lambda_{y}\right)$ is a constant vector and $\lambda_{x}>0, \lambda_{y}>0$ will be assumed in this section (other cases are detailed in Appendix B). The exact solution of (53), from some initial condition $q\left(x^{\prime}, y^{\prime}, t\right)$ is expressed in global coordinate:

$$
q\left(x^{\prime}, y^{\prime}, t+\Delta t\right)=q\left(x^{\prime}-\lambda_{x} \Delta t, y^{\prime}-\lambda_{y} \Delta t, t\right)
$$

and transformed in local coordinate:

$$
q(x, y, t+\Delta t)=q\left(x-2 c_{x}, y-2 c_{y}, t\right)
$$

Where $c_{x}$ and $c_{y}$ are defined as:

$$
\begin{gathered}
c_{x}=\frac{\left|\lambda_{x}\right| \Delta t}{\Delta x^{\prime}} \\
c_{y}=\frac{\left|\lambda_{y}\right| \Delta t}{\Delta y^{\prime}}
\end{gathered}
$$

Starting from the initial conditions $q_{i, j}(x, y, t)$ as in $(52)$ the exact solution of the transport equation at time $t+\Delta t$ can be expressed as:

$$
\widetilde{q}_{i, j}(x, y, t+\Delta t)=
$$




$$
\begin{cases}q_{i-1, j-1}\left(x+2-2 c_{x}, y+2-2 c_{y}, t\right) & \text { if }-1<x<2 c_{x}-1, \quad-1<y<-1+2 c_{y} \\ q_{i-1, j}\left(x+2-2 c_{x}, y-2 c_{y}, t\right) & \text { if }-1<x<2 c_{x}-1, \quad 2 c_{y}-1<y<1 \\ q_{i, j-1}\left(x-2 c_{x}, y+2-2 c_{y}, t\right) & \text { if } 2 c_{x}-1<x<1, \quad-1<y<-1+2 c_{y} \\ q_{i, j}\left(x-2 c_{x}, y-2 c_{y}, t\right) & \text { if } 2 c_{x}-1<x<1, \quad 2 c_{y}-1<y<1\end{cases}
$$

which is valid if $\Delta t$ is so that:

$$
\max \left(c_{x}, c_{y}\right)<1
$$

A functional approximation of (58) is performed in order to reach the updated set of coefficients in the grid cell.

$$
q_{i, j}(x, y, t+\Delta t)=\sum_{m=1}^{N} \sum_{n=1}^{N}{ }^{m, n} q_{i, j}^{t+\Delta t} P_{m}(x) P_{n}(y)
$$

with the coefficients:

$$
{ }^{m, n} q_{i, j}^{t+\Delta t}=\frac{(2 m-1)(2 n-1)}{4} \int_{-1}^{1} \int_{-1}^{1} \widetilde{q}_{i, j}(x, y, t+\Delta t) P_{m}(x) P_{n}(y) d x d y
$$

\subsection{Updating scheme in two dimensions}

The exact solution of the integrals in (61) has been used to construct the numerical scheme in two dimensions:

$$
\begin{aligned}
{ }^{m, n} q_{i, j}^{t+\Delta t}= & \sum_{k=1}^{N} \sum_{l=1}^{N}{ }^{k, l} q_{i-1, j-1}^{t} L_{m, k}\left(c_{x}\right) L_{n, l}\left(c_{y}\right)+\sum_{k=1}^{N} \sum_{l=1}^{N}{ }^{k, l} q_{i, j-1}^{t} R_{m, k}\left(c_{x}\right) L_{n, l}\left(c_{y}\right) \\
& +\sum_{k=1}^{N} \sum_{l=1}^{N}{ }^{k, l} q_{i-1, j}^{t} L_{m, k}\left(c_{x}\right) R_{n, l}\left(c_{y}\right)+\sum_{k=1}^{N} \sum_{l=1}^{N}{ }^{k, l} q_{i, j}^{t} R_{m, k}\left(c_{x}\right) R_{n, l}\left(c_{y}\right)
\end{aligned}
$$

where the matrix $L$ and $R$ are, as defined previously in the one dimensional scheme, (34) and (35) respectively.

This numerical scheme is based on an approximation of the exact solution of the transport equation. Arising from the pure advective property, the classical $C F L$ stability 
condition applies (59), being the numerical stability controlled by the most restrictive of both $c_{x}$ and $c_{y}$. It is important to stress that the stability condition of the CFA method, as derived, is independent of the order of accuracy and the number of spatial dimensions involved.

In a simulation with $\Delta t$ and $\Delta x$ of constant size, matrices $L$ and $R$ in (62) are constant. Taking into account that the maximum time step allowed is independent of the order and the number of spatial dimensions, the total computational cost of the CFA method grows at a rate of $D N^{2 D}$, being $D$ the number of spatial dimensions and $N$ the order of accuracy.

\section{$5 \quad$ Numerical tests in two dimensions}

\section{$5.1 \quad$ Test 5}

A first set of simulations are presented in order to numerically evaluate the order of accuracy of the different approximations. The two-dimensional transport of a Gaussian function:

$$
q\left(x^{\prime}, y^{\prime}\right)=e^{-\left(\left(x^{\prime}-1\right)^{2}+\left(y^{\prime}-1\right)^{2}\right) / 0.05}
$$

in a square domain $x^{\prime} \in[0,50], y^{\prime} \in[0,50]$ by means of a unit advection velocity at different directions in the plane, $\lambda_{x}=\cos (\alpha), \lambda_{y}=\sin (\alpha)$, will be used for this purpose. Here $\alpha$ is the angle formed by the advection velocity vector and the $x$ axis. The reason to test the accuracy at different advection angles is to estimate the ability of the method to produce a numerical solution that is independent of the grid alignment. The method as presented, when applied in $2 \mathrm{D}$ does not perform independent updating steps in every direction, instead it contains crossed terms. Figure 12 shows the description of the test case. Simulation time of $t=40$ was used with a fixed time step base on the condition $\max \left(c_{x}, c_{y}\right)=0.95$. Simulations with the CFA method of orders of accuracy 1, 2 and 3 on grids of different degree of refinement will be considered. The results will be compared using the $L_{1}$ norm of the error relative to the exact solution: 


$$
L_{1}=\frac{1}{4 M^{2}} \sum_{i=1}^{M} \sum_{j=1}^{M} \int_{-1}^{1} \int_{-1}^{1}\left|q_{i, j}(x, y)-q_{\text {Exact }}(x, y)\right| d x d y
$$

where $M^{2}$ represents the cell number and the integrals in (64) are numerically computed.

The error norm for the CFA scheme of orders of accuracy 1, 2 and 3 and for the angles $\alpha=0^{\circ}, 22.5^{\circ}$ and $45^{\circ}$ is plotted in logarithmic scale ordered by angles in Figure 13 showing that the theoretical convergence is reached independently of the advection angle.

\subsection{Test 6}

As an efficiency test case, Test 5 is reconsidered for a relative comparison of the computational cost involved by the different orders of approximation. We are interested in the optimal combination of grid refinement and order of accuracy able to provide a desired maximum error at the minimum computational cost. Figure 15 displays the shape of the numerical solutions corresponding to values of $\log \left(L_{1}\right)=-4.0$ and $\log \left(L_{1}\right)=-4.5$ as compared to the exact solution in order to state the acceptable size of this error in practical applications. The error of the results and the computational time of the schemes of orders of accuracy 1, 2 and 3 for angles $\alpha=0^{\circ}, 22.5^{\circ}$ and $45^{\circ}$ is plotted ordered by angle in Figure 16 and grouped by order in Figure 17.

Figure 16 shows that the same error can be achieved using schemes of different order of accuracy over different grids and using different computational time. It is worth noting that a first order method with increased grid refinement is the less efficient option. Figure 16 is also useful to see that starting from the error generated by a first order scheme on a given computational mesh, better quality solutions can be obtained at the same computational costs by increasing the order of accuracy of the scheme and coarsening the grid.

\subsection{Test 7}

This test is intended to focus on the angular dependence of the numerical results of the schemes of different order of accuracy. For that purpose, the computational domain and 
the initial conditions of Test. 5 are reconsidered. A simulation time of $t=8$, a time step according to the condition $\max \left(c_{x}, c_{y}\right)=0.95$ and a cell size of $\Delta x=0.6$ where used in all cases. The schemes of orders of accuracy 1,2 and 3 have been used to simulate the advection along the 91 directions corresponding to the angles ranging from $0^{\circ}$ to $90^{\circ}$. Figure 18 is a polar plot of the error defined as (64). It can be observed that the three methods perform better at the angles $0^{\circ}, 45^{\circ}$ and $90^{\circ}$ due to the Cartesian based design of the advection schemes (62). As the order of accuracy of the method increases new preferential directions appear. However the convergence of the error of all the orders of accuracy, independently of the advection angle, has already been proved.

\subsection{Test 8}

The last example has been chosen to show the possibility offered by this family of methods to obtain numerical solutions of increasing accuracy at a given computational cost using less grid cells. A discontinuous initial profile is defined in a square domain $x^{\prime} \in[0,12]$, $y^{\prime} \in[0,12]$ as

$$
q\left(x^{\prime}, 0\right)= \begin{cases}0 & \text { if }\left(x^{\prime}-1\right)^{2}+\left(y^{\prime}-1\right)^{2}>1 \\ 0 & \text { if } y^{\prime}>1 \text { and } x^{\prime}>0.9 \text { and } x^{\prime}<1.1 \\ 1 & \text { otherwise }\end{cases}
$$

The CFA schemes of orders of accuracy 1,2 and 3 have been used to simulate the advection of this shape with and speed of unit norm at an angle $\alpha=45^{\circ}$ during time $t=8$. The time step has been chosen according to $\max \left(c_{x}, c_{y}\right)=0.5$.

Figure 19 a) is a contour plot of the exact solution at any time. Figure $19 \mathrm{~b}$ ) is a contour plot of the numerical results obtained by the CFA first order method using a $150 \times 150$ cell grid at $t=8$. Figure $19 \mathrm{c}$ ) and Figure $19 \mathrm{~d}$ ) correspond to the solutions by the CFA second order and CFA third order schemes respectively at the same time. The second order solution has been computed on a $60 x 60$ cell grid and the third order solution on a $35 x 35$ cell grid with a computational cost equivalent to the first order solution on a $150 x 150$ cell grid. The $L_{1}$ error norm of the schemes of the three orders on those grids 
are shown in Table 1.

\section{Conclusions}

A new approximation well suited for high order advection simulation in multidimensional problems has been presented. The scheme is explicit and based on a single updating step. Piecewise polynomial spatial discretization using Legendre polynomials provides the required spatial accuracy and the subgrid information. The updating scheme is built from the functional approximation of the exact solution of the advection equation and a direct evaluation of the resulting integrals.

The numerical details for the $1 \mathrm{D}$ and $2 \mathrm{D}$ cases have been provided and the schemes have been validated using a set of numerical experiments. Some of the test cases have been oriented to the convergence analysis of the schemes of different order of accuracy both in $1 \mathrm{D}$ and in 2D with an special interest in the computational efficiency of the different options. The results from the schemes from $1^{\text {st }}$ to $5^{\text {th }}$ order of accuracy have been presented in 1D test cases showing that, in general, given a desired maximum error, there is a scheme of a certain order of accuracy able to provide the solution at the lowest cost. It is also worth remarking that if when starting by a first order approach the numerical error is excessive, refining the grid is never the best option to reduce the error. It is also true that for longer simulations (either in larger domains) higher order approximations gain in relative efficiency.

The numerical performance of the CFA second order scheme has been compared with that of the Lax-Wendroff and second order upwind schemes showing that, although the three second order schemes produce oscillations near discontinuities, the CFA scheme, using the same the same stored information as the other two methods, provides a more accurate and less oscillatory solution using a smaller computational time. A slope limiting procedure to reduce the oscillations has been outlined and presented as efficient in this case.

The 1D test cases have been also useful to show the ability of the CFA family of schemes to generate and propagate information at the subgrid scale. An example displaying this 
kind of results obtained by the CFA method of order of accuracy 20 has been presented.

The 2D test cases have been useful to analyze the multidimensional character of the schemes. For that purpose, the advection along different directions in a given Cartesian mesh has been studied using the CFA method of order of accuracy 1, 2 and 3. The results show that the convergence is achieved by all the orders with independence of the advection direction. As the order increases, more preferential directions appear. From the efficiency point of view, the conclusion is the same as in 1D, that is, a first order method with increased mesh refinement is not the most efficient option. On the contrary, better quality solutions can be obtained at the same cost by increasing the order of the scheme and coarsening the grid. To emphasize this point, a test case has been included where, given a desirable computational time, different grids and orders of accuracy have been used showing that it is possible to use methods of high order to obtain results with less error more efficiently than first order methods.

\section{Acknowledgements}

This research work has been partially funded by the Spanish Ministry of Science and Education under research project CGL2005-07059-C02-02.

\section{A Derivation of the 1D numerical scheme}

The steps necessary to develop the updating scheme are next presented for the $1 \mathrm{D}$ case with advection velocity $\lambda>0$. The starting point is the expression of the exact transported solution at grid cell $i$ and time $t+\Delta t$ :

$$
\widetilde{q}_{i}(x, t+\Delta t)= \begin{cases}q_{i-1}(x+2-2 c, t) & \text { if }-1<x<2 c-1 \\ q_{i}(x-2 c, t) & \text { if } 2 c-1<x<1\end{cases}
$$

A conservative functional approximation of (66) is written as:

$$
q_{i}(x, t+\Delta t)=\sum_{n=1}^{N}{ }^{n} q_{i}^{t+\Delta t} P_{n}(x) \simeq \widetilde{q}_{i}(x, t+\Delta t)
$$


where the coefficients are calculated as:

$$
{ }^{n} q_{i}^{t+\Delta t}=\frac{2 n-1}{2} \int_{-1}^{1} \widetilde{q}_{i}(x, t+\Delta t) P_{n}(x) d x
$$

The integral in (68) is transformed by inserting (66):

$$
\begin{gathered}
{ }^{n} q_{i}^{t+\Delta t}=\frac{2 n-1}{2} \int_{-1}^{2 c-1} q_{i-1}(x+2-2 c, t) P_{n}(x) d x \\
+\frac{2 n-1}{2} \int_{2 c-1}^{1} q_{i}(x-2 c, t) P_{n}(x) d x
\end{gathered}
$$

The two terms in the right hand side of (69) are denoted $S_{1}$ and $S_{2}$ and we proceed to their calculation.

$$
S_{1}=\frac{2 n-1}{2} \int_{-1}^{2 c-1} q_{i-1}(x+2-2 c, t) P_{n}(x) d x
$$

First, a change of variable is applied in order to adapt the integration domain to the domain of orthogonality of the Legendre polynomials.

$$
S_{1}=\frac{2 n-1}{2} \int_{-1}^{1} q_{i-1}(c x-c+1, t) P_{n}(c x+c-1) c d x
$$

Then, the distribution $q_{i-1}(x, t)$ is replaced within the integral by its functional representation as in (21):

$$
S_{1}=\sum_{j=1}^{N}{ }^{j} q_{i-1}^{t} \frac{2 n-1}{2} \int_{-1}^{1} P_{j}(c x-c+1) P_{n}(c x+c-1) c d x
$$

The Legendre polynomials $P_{n}(a x+b)$ are expressed as linear combination of the original $P_{n}(x)$ using (37) so that:

$$
S_{1}=\sum_{j=1}^{N}{ }^{j} q_{i-1}^{t} \frac{2 n-1}{2} \sum_{k=1}^{N} \sum_{l=1}^{N} c A_{j, k}(c, 1-c) A_{n, l}(c, c-1) \int_{-1}^{1} P_{k}(x) P_{l}(x) d x
$$

The orthogonality properties of the Legendre polynomials (7) can be used to simplify (73) leading to: 


$$
S_{1}=\sum_{j=1}^{N}{ }^{j} q_{i-1}^{t} \frac{2 n-1}{2} \sum_{k=1}^{N} c \frac{2}{2 k-1} A_{j, k}(c, 1-c) A_{n, k}(c, c-1)
$$

And finally a compact expression can be obtained by defining matrices $T$ and $L$ according to (36) and (34) respectively as follows:

$$
S_{1}=\sum_{j=1}^{N}{ }^{j} q_{i-1}^{t} T_{n, j}(c, 1-c)=\sum_{j=1}^{N}{ }^{j} q_{i-1}^{t} L_{n, j}(c)
$$

Next, the calculation of the second term $S_{2}$ is presented:

$$
S_{2}=\frac{2 n-1}{2} \int_{2 c-1}^{1} q_{i}(x-2 c, t) P_{n}(x) d x
$$

A similar change of variable can be made leading to:

$$
S_{2}=\frac{2 n-1}{2} \int_{-1}^{1} q_{i}((1-c) x-c, t) P_{n}((1-c) x+c)(1-c) d x
$$

Then, the functional approximation of the distribution $q_{i}(x, t)$ gives:

$$
S_{2}=\sum_{j=1}^{N}{ }^{j} q_{i}^{t} \frac{2 n-1}{2} \int_{-1}^{1} P_{j}((1-c) x-c) P_{n}((1-c) x+c)(1-c) d x
$$

Transforming the Legendre polynomials of the form $P_{n}(a x+b)$ into linear combinations of the original $P_{n}(x)$ as in (37):

$$
S_{2}=\sum_{j=1}^{N}{ }^{j} q_{i}^{t} \frac{2 n-1}{2} \sum_{k=1}^{N} \sum_{l=1}^{N}(1-c) A_{j, k}(1-c,-c) A_{n, l}(1-c, c) \int_{-1}^{1} P_{k}(x) P_{l}(x) d x
$$

Then, using the Legendre polynomial properties, (79) can be simplified to:

$$
S_{2}=\sum_{j=1}^{N}{ }^{j} q_{i}^{t} \frac{2 n-1}{2} \sum_{k=1}^{N}(1-c) \frac{2}{2 k-1} A_{j, k}(1-c,-c) A_{n, k}(1-c, c)
$$


The compact notation is based again on the definition of matrices $T$ and $R$ according to $(36)$ and $(35)$ :

$$
S_{2}=\sum_{j=1}^{N}{ }^{j} q_{i}^{t} T_{n, j}(1-c,-c)=\sum_{j=1}^{N}{ }^{j} q_{i}^{t} R_{n, j}(c)
$$

The final expression is built by using both results and leads to the formulation of the numerical scheme:

$$
{ }^{n} q_{i}^{t+\Delta t}=\sum_{j=1}^{N}{ }^{j} q_{i-1}^{t} L_{n, j}(c)+\sum_{j=1}^{N}{ }^{j} q_{i}^{t} R_{n, j}(c)
$$

\section{B Formulation of the method in 2D}

The exact solution of the 2D transport equation at time $t+\Delta t$ is outlined considering all the possible signs of the advection speed:

- $\lambda_{x}>0, \lambda_{y}>0$ :

$$
\begin{gathered}
\widetilde{q}_{i, j}(x, y, t+\Delta t)= \\
\begin{cases}q_{i-1, j-1}\left(x+2-2 c_{x}, y+2-2 c_{y}, t\right) & \text { if }-1<x<2 c_{x}-1, \quad-1<y<-1+2 c_{y} \\
q_{i-1, j}\left(x+2-2 c_{x}, y-2 c_{y}, t\right) & \text { if }-1<x<2 c_{x}-1, \quad 2 c_{y}-1<y<1 \\
q_{i, j-1}\left(x-2 c_{x}, y+2-2 c_{y}, t\right) & \text { if } 2 c_{x}-1<x<1, \quad-1<y<-1+2 c_{y} \\
q_{i, j}\left(x-2 c_{x}, y-2 c_{y}, t\right) & \text { if } 2 c_{x}-1<x<1, \quad 2 c_{y}-1<y<1\end{cases}
\end{gathered}
$$

- $\lambda_{x}<0, \lambda_{y}>0$ :

$$
\widetilde{q}_{i, j}(x, y, t+\Delta t)=
$$




$$
\begin{cases}q_{i+1, j-1}\left(x-2+2 c_{x}, y+2-2 c_{y}, t\right) & \text { if } 1-2 c_{x}<x<1, \quad-1<y<-1+2 c_{y} \\ q_{i+1, j}\left(x-2+2 c_{x}, y-2 c_{y}, t\right) & \text { if } 1-2 c_{x}<x<1, \quad 2 c_{y}-1<y<1 \\ q_{i, j-1}\left(x+2 c_{x}, y+2-2 c_{y}, t\right) & \text { if }-1<x<1-2 c_{x}, \quad-1<y<-1+2 c_{y} \\ q_{i, j}\left(x+2 c_{x}, y-2 c_{y}, t\right) & \text { if }-1<x<1-2 c_{x}, \quad 2 c_{y}-1<y<1\end{cases}
$$

- $\lambda_{x}>0, \lambda_{y}<0$

$$
\begin{gathered}
\widetilde{q}_{i, j}(x, y, t+\Delta t)= \\
\begin{cases}q_{i-1, j+1}\left(x+2-2 c_{x}, y-2+2 c_{y}, t\right) & \text { if }-1<x<2 c_{x}-1, \quad 1-2 c_{y}<y<1 \\
q_{i-1, j}\left(x+2-2 c_{x}, y+2 c_{y}, t\right) & \text { if }-1<x<2 c_{x}-1, \quad-1<y<1-2 c_{y} \\
q_{i, j+1}\left(x-2 c_{x}, y-2+2 c_{y}, t\right) & \text { if } 2 c_{x}-1<x<1, \quad 1-2 c_{y}<y<1 \\
q_{i, j}\left(x-2 c_{x}, y+2 c_{y}, t\right) & \text { if } 2 c_{x}-1<x<1,-1<y<1-2 c_{y}\end{cases}
\end{gathered}
$$

- $\lambda_{x}<0, \lambda_{y}<0$

$$
\begin{gathered}
\widetilde{q}_{i, j}(x, y, t+\Delta t)= \\
\begin{cases}q_{i+1, j+1}\left(x-2+2 c_{x}, y-2+2 c_{y}, t\right) & \text { if } 1-2 c_{x}<x<1, \quad 1-2 c_{y}<y<1 \\
q_{i+1, j}\left(x-2+2 c_{x}, y+2 c_{y}, t\right) & \text { if } 1-2 c_{x}<x<1, \quad-1<y<1-2 c_{y} \\
q_{i, j+1}\left(x+2 c_{x}, y-2+2 c_{y}, t\right) & \text { if }-1<x<1-2 c_{x}, \quad 1-2 c_{y}<y<1 \\
q_{i, j}\left(x+2 c_{x}, y+2 c_{y}, t\right) & \text { if }-1<x<1-2 c_{x}, \quad-1<y<1-2 c_{y}\end{cases}
\end{gathered}
$$

The resulting 2D numerical scheme, considering all the possible signs of the advection speed is:

- $\lambda_{x}>0, \lambda_{y}>0$ 


$$
\begin{aligned}
{ }^{m, n} q_{i, j}^{t+\Delta t}= & \sum_{k=1}^{N} \sum_{l=1}^{N}{ }^{k, l} q_{i-1, j-1}^{t} L_{m, k}\left(c_{x}\right) L_{n, l}\left(c_{y}\right) \\
& +\sum_{k=1}^{N} \sum_{l=1}^{N}{ }^{k, l} q_{i, j-1}^{t} R_{m, k}\left(c_{x}\right) L_{n, l}\left(c_{y}\right) \\
& +\sum_{k=1}^{N} \sum_{l=1}^{N}{ }^{k, l} q_{i-1, j}^{t} L_{m, k}\left(c_{x}\right) R_{n, l}\left(c_{y}\right) \\
& +\sum_{k=1}^{N} \sum_{l=1}^{N}{ }^{k, l} q_{i, j}^{t} R_{m, k}\left(c_{x}\right) R_{n, l}\left(c_{y}\right)
\end{aligned}
$$

- $\lambda_{x}<0, \lambda_{y}>0$

$$
\begin{aligned}
{ }^{m, n} q_{i, j}^{t+\Delta t}= & \sum_{k=1}^{N} \sum_{l=1}^{N}{ }^{k, l} q_{i+1, j-1}^{t} R_{m, k}\left(1-c_{x}\right) L_{n, l}\left(c_{y}\right) \\
& +\sum_{k=1}^{N} \sum_{l=1}^{N}{ }^{k, l} q_{i, j-1}^{t} L_{m, k}\left(1-c_{x}\right) L_{n, l}\left(c_{y}\right) \\
& +\sum_{k=1}^{N} \sum_{l=1}^{N}{ }^{k, l} q_{i+1, j}^{t} R_{m, k}\left(1-c_{x}\right) R_{n, l}\left(c_{y}\right) \\
& +\sum_{k=1}^{N} \sum_{l=1}^{N}{ }^{k, l} q_{i, j}^{t} L_{m, k}\left(1-c_{x}\right) R_{n, l}\left(c_{y}\right)
\end{aligned}
$$

- $\lambda_{x}>0, \lambda_{y}<0$

$$
\begin{aligned}
{ }^{m, n} q_{i, j}^{t+\Delta t}= & \sum_{k=1}^{N} \sum_{l=1}^{N}{ }^{k, l} q_{i-1, j+1}^{t} L_{m, k}\left(c_{x}\right) R_{n, l}\left(1-c_{y}\right) \\
& +\sum_{k=1}^{N} \sum_{l=1}^{N}{ }^{k, l} q_{i, j+1}^{t} R_{m, k}\left(c_{x}\right) R_{n, l}\left(1-c_{y}\right) \\
& +\sum_{k=1}^{N} \sum_{l=1}^{N}{ }^{k, l} q_{i-1, j}^{t} L_{m, k}\left(c_{x}\right) L_{n, l}\left(1-c_{y}\right) \\
& +\sum_{k=1}^{N} \sum_{l=1}^{N}{ }^{k, l} q_{i, j}^{t} R_{m, k}\left(c_{x}\right) L_{n, l}\left(1-c_{y}\right)
\end{aligned}
$$

- $\lambda_{x}<0, \lambda_{y}<0$ 


$$
\begin{aligned}
{ }^{m, n} q_{i, j}^{t+\Delta t}= & \sum_{k=1}^{N} \sum_{l=1}^{N}{ }^{k, l} q_{i+1, j+1}^{t} R_{m, k}\left(1-c_{x}\right) R_{n, l}\left(1-c_{y}\right) \\
& +\sum_{k=1}^{N} \sum_{l=1}^{N}{ }^{k, l} q_{i, j+1}^{t} L_{m, k}\left(1-c_{x}\right) R_{n, l}\left(1-c_{y}\right) \\
& +\sum_{k=1}^{N} \sum_{l=1}^{N}{ }^{k, l} q_{i+1, j}^{t} R_{m, k}\left(1-c_{x}\right) L_{n, l}\left(1-c_{y}\right) \\
& +\sum_{k=1}^{N} \sum_{l=1}^{N}{ }^{k, l} q_{i, j}^{t} L_{m, k}\left(1-c_{x}\right) L_{n, l}\left(1-c_{y}\right)
\end{aligned}
$$

\section{References}

[1] M. Ben-Artzi and J. Falcovitz. A second-order godunov-type scheme forcompressible fluid dynamics. Journal of Computational Physics, 55:1-32, 1984.

[2] J. C. Butcher. The numerical analysis of ordinary differential equations: RungeKutta and general linear methods. Wiley-Interscience, New York, NY, USA, 1987.

[3] Michael Dumbser, Dinshaw S. Balsara, Eleuterio F. Toro, and Claus-Dieter Munz. A unified framework for the construction of one-step finite volume and discontinuous galerkin schemes on unstructured meshes. J. Comput. Phys., 227(18):8209-8253, 2008.

[4] S. K. Godunov. A finite difference method for the computation of discontinious solutions of the equation of fluid dynamics. Mat. Sb., 47:357âĂŞ393, 1959.

[5] S. Gottlieb and C. W. Shu. Total variation diminishing runge-kutta schemes. Mathematics of Computation, 67:73-85, 1998.

[6] A. Harten, P. D. Lax, and B. van Leer. On upstreaming differencing and godunovtype schemes for hyperbolic conservation laws. SIAM Rev., 25:35-61, 1983.

[7] CH. Hirch. Numerical computation of internal and external flows. Vol. 1: Fundamentals of numerical discretization. Wiley, 1989.

[8] A. J. Jerri. The Gibbs Phenomenon in Fourier Analysis, Splines and Wavelet Approximations. Kluwer, 1998. 
[9] G.-S. Jiang and C.W. Shu. Efficient implementation of weighted eno schemes. Journal of Computational Physics, 126:202-228, 1996.

[10] R. J. LeVeque. Finite Volume Methods for Hyperbolic Problems. Cambridge University Press, 2002.

[11] P. L. Roe. Approximate riemann solvers, parameter vectors, and difference schemes. Journal of Computational Physics, 43:357-372, 1981.

[12] J. C. Rutherford. River Mixing. John Wiley Sons Ltd, 1994.

[13] V.A. Titarev and E.F. Toro. Ader: Arbitrary high order godunov approach. Journal of Scientific Computing, 17:609-618, 2002.

[14] E. F. Toro, R. C. Millington, and L. A. M. Nejad. Towards very high order Godunov schemes. Kluwer/Plenum Academic Publishers, 2001.

[15] E. F. Toro and V. A. Titarev. Ader schemes for scalar non-linear hyperbolic conservation laws with source terms in three-space dimensions. J. Comput. Phys., 202(1):196215,2005 .

[16] E. F. Toro and V. A. Titarev. Tvd fluxes for the high-order ader schemes. J. Sci. Comput., 24(3):285-309, 2005.

[17] B. van Leer. Towards the ultimate conservative difference scheme v: A second order sequel to godunovs method. Journal of Computational Physics, 32:101-136, 1979.

[18] Feng Xiao and Takashi Yabe. Completely conservative and oscillationless semilagrangian schemes for advection transportation. Journal of Computational Physics, $170(2): 498-522,2001$. 


\begin{tabular}{|c|c|c|c|}
\hline & Order 1 & Order 2 & Order 3 \\
\hline Cells & $150 \times 150$ & $60 \times 60$ & $35 \times 35$ \\
\hline $\mathbf{L}_{\mathbf{1}}$ & $1.73 \cdot 10^{-2}$ & $6.80 \cdot 10^{-3}$ & $5.23 \cdot 10^{-3}$ \\
\hline
\end{tabular}

Table 1: $L_{1}$ errors produced by the CFA schemes of orders of accuracy 1,2 and 3 using different computational meshes in Test 8 . The three simulations are achieved in the same computational time. 
a) Exact initial condition

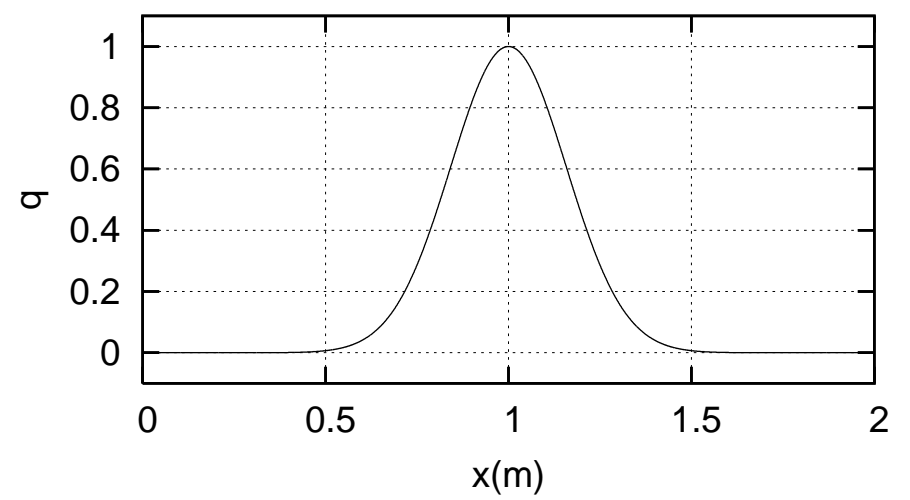

Exact Initial Condition

b) Approximations of the initial condition

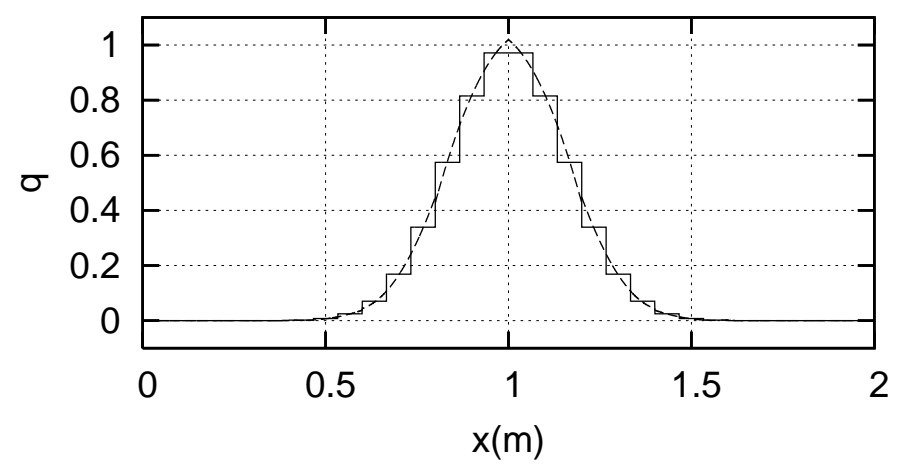

Order $=1, M=30$ cells

Order $=3, M=10$ cells

Figure 1: Initial distribution a) and approximations of first and third order using the same amount of information b). 
a) Initial conditions. Time=t

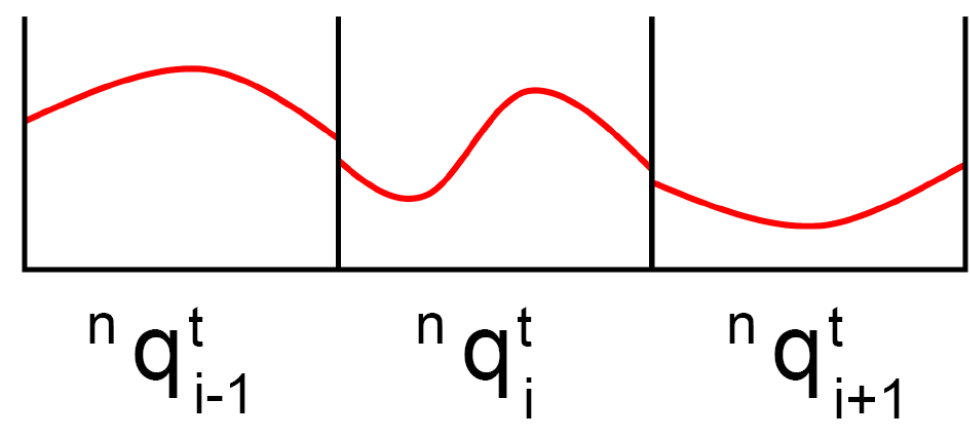

b) Exact solution. Time $=t+\Delta t$

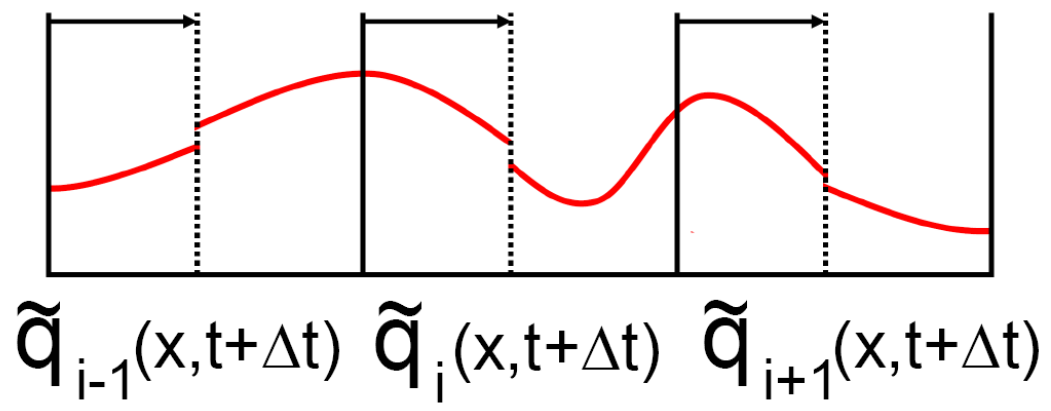

c) Final approximation. Time $=t+\Delta t$

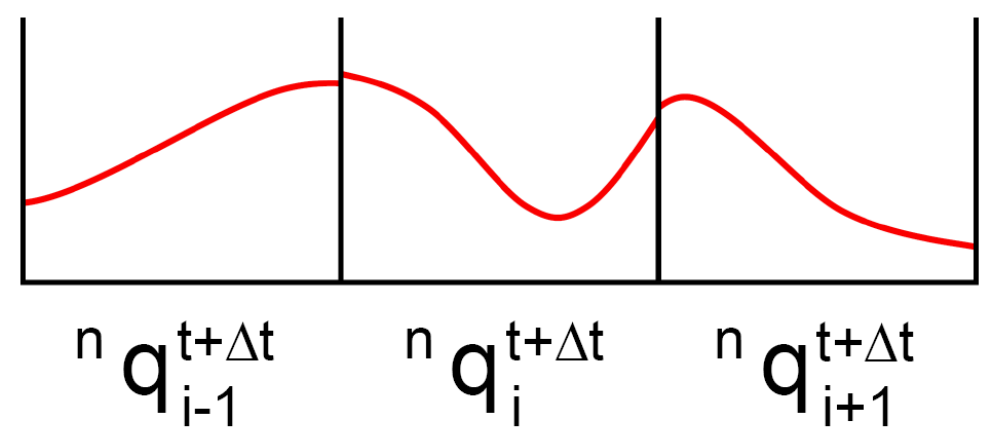

Figure 2: Description of the steps in the CFA method. Starting from the initial condition a), the transported exact solution at the new time step is obtained b) and approximated c). 


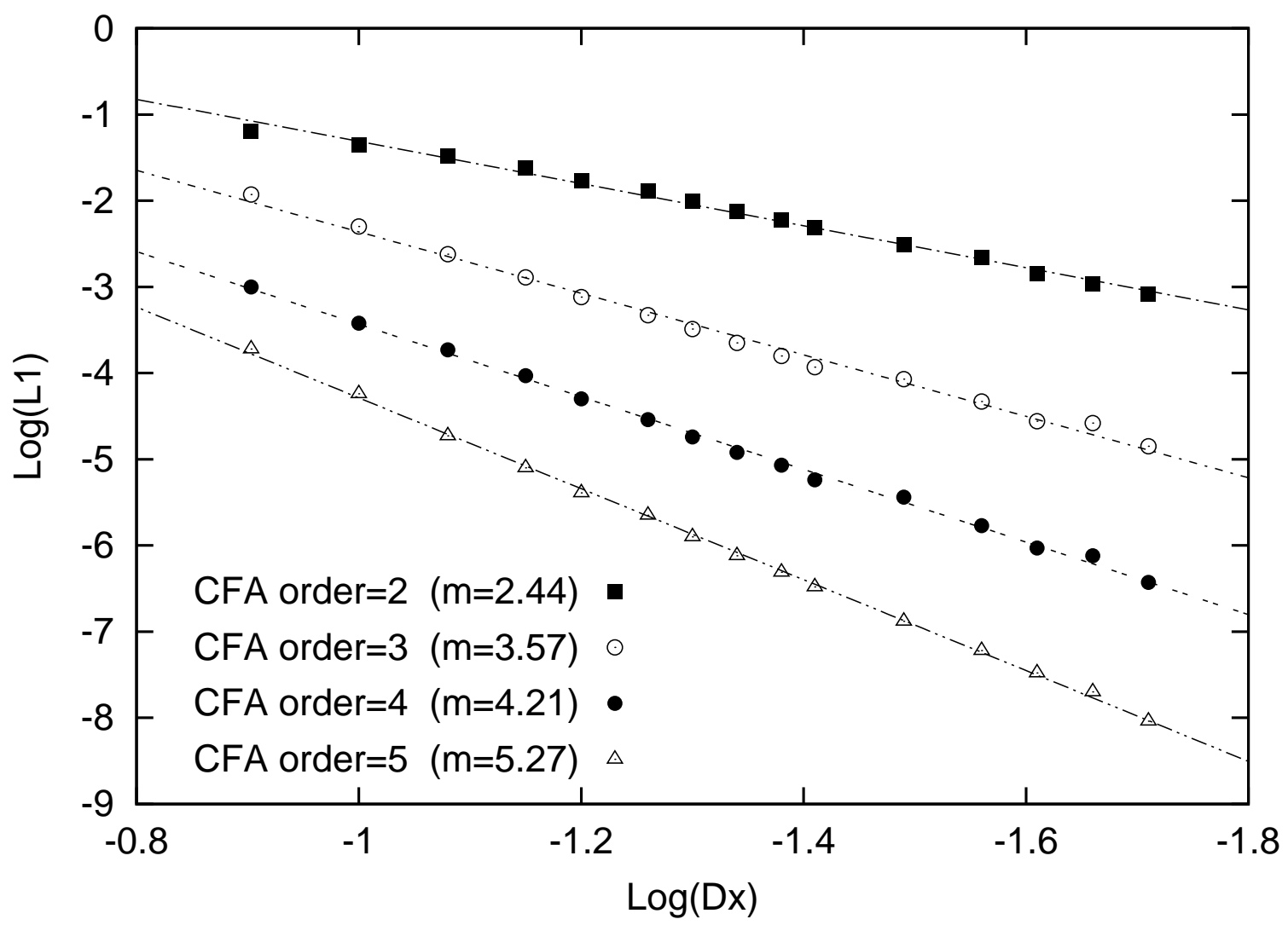

Figure 3: Error convergence of the CFA methods of order of accuracy 2, 3, 4 and 5 in the numerical test 1 . 


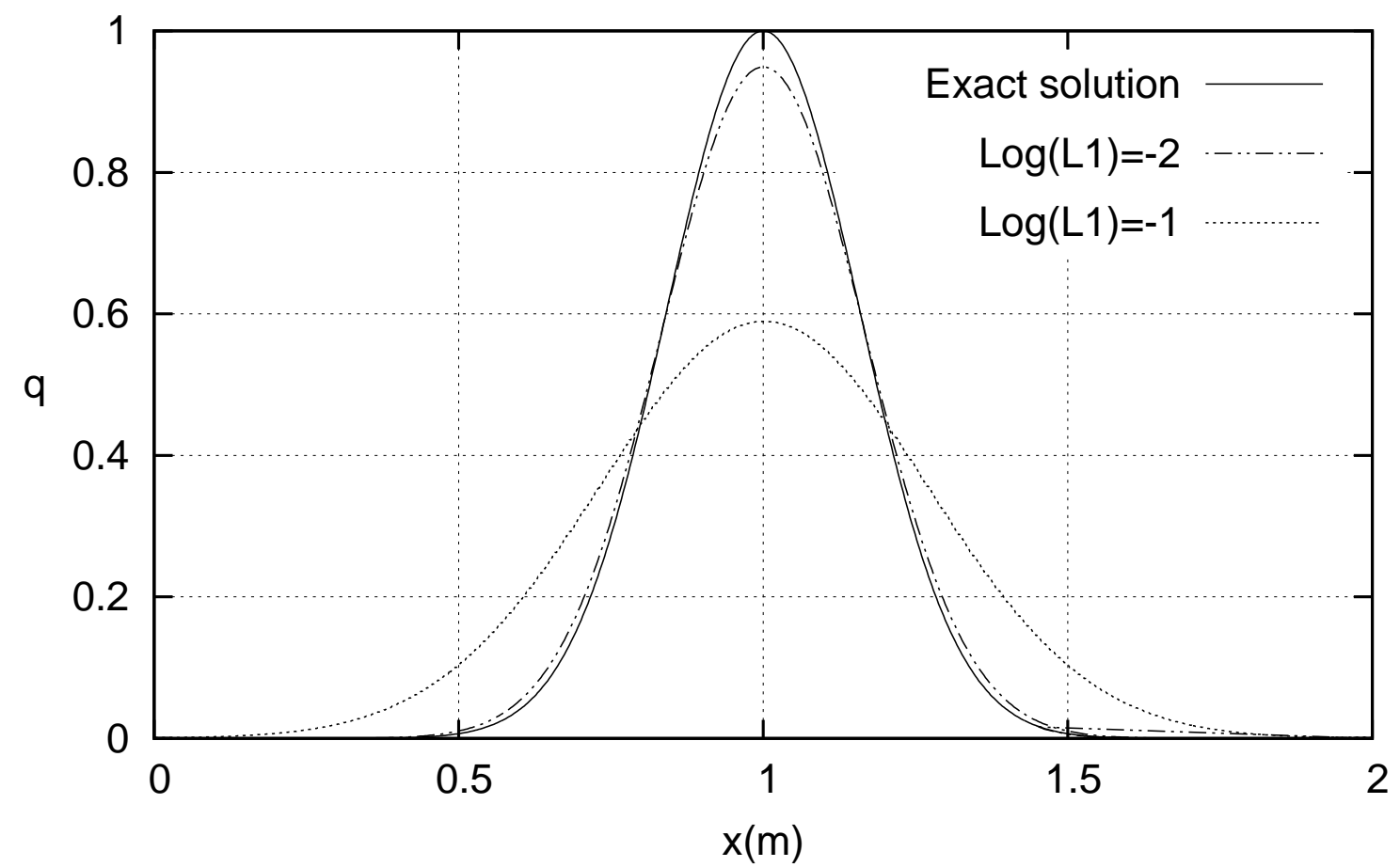

Figure 4: Shape of the numerical solutions corresponding to values of $\log \left(L_{1}\right)=-1$ and $\log \left(L_{1}\right)=-2$ compared to the exact solution in order to visualize the size of the $L_{1}$ error in Test case 2 . 
a) End time $=20 \mathrm{~s}$

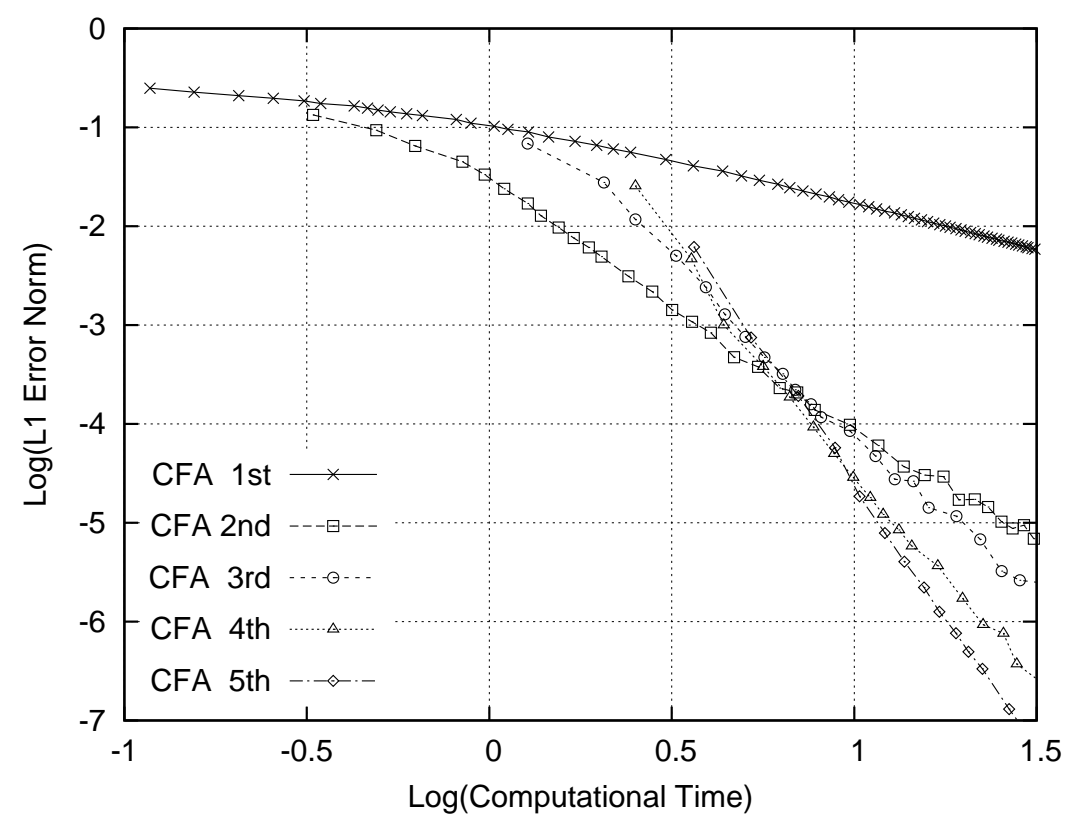

b) End time $=200 \mathrm{~s}$

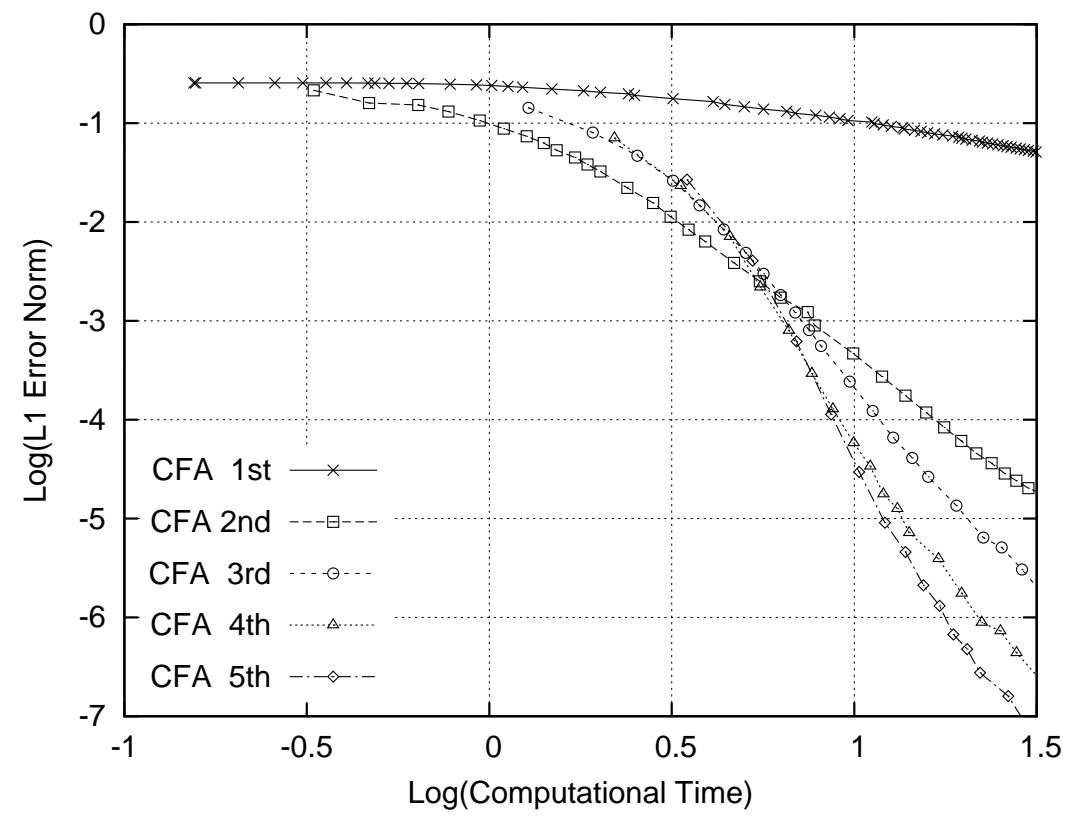

Figure 5: $L_{1}$ error norm produced by the CFA schemes of orders of accuracy 1, 2, 3, 4 and 5 as a function of the computational time used in numerical Test 2. 
a) CFA Second Order
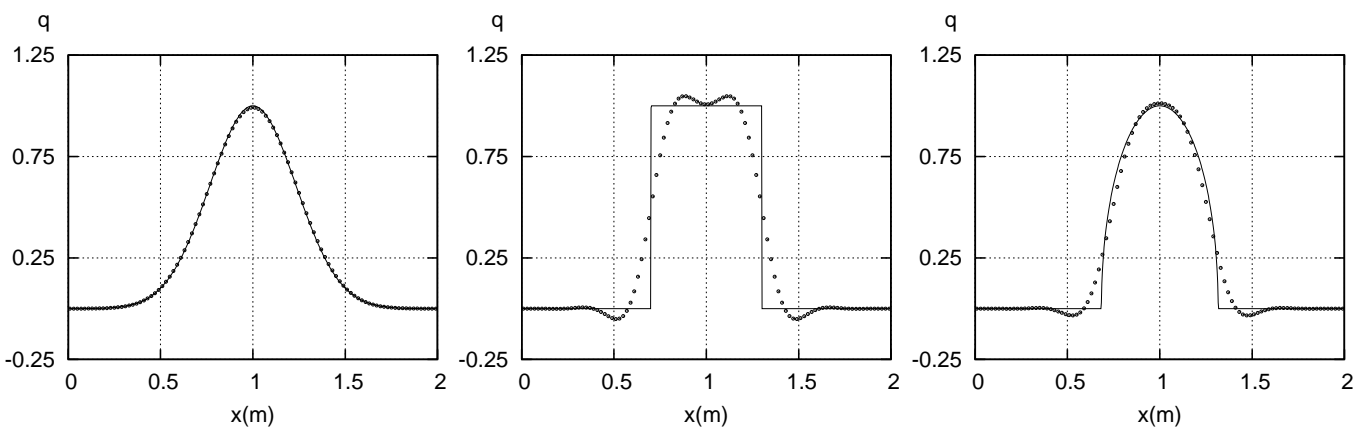

b) Lax-Wendroff
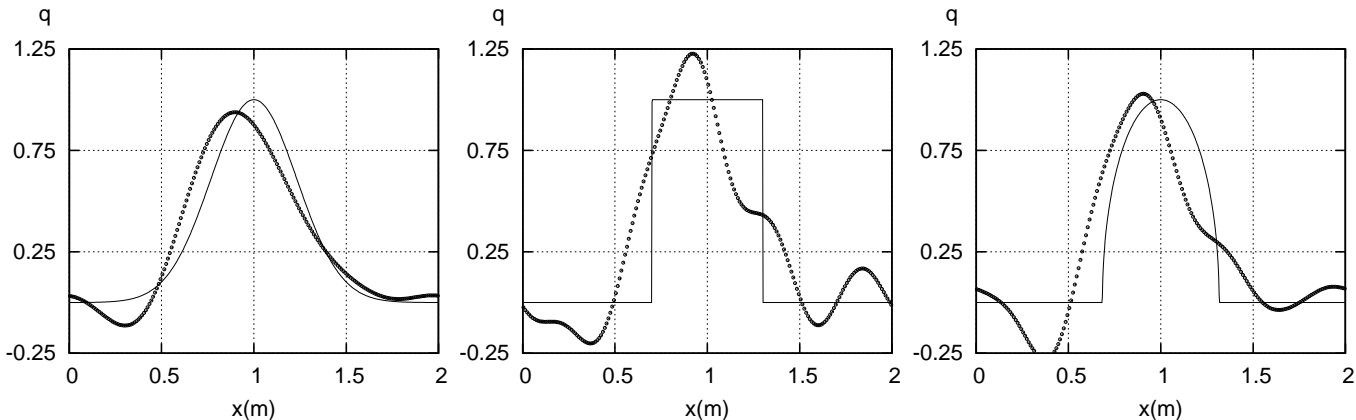

c) Upwind Second Order
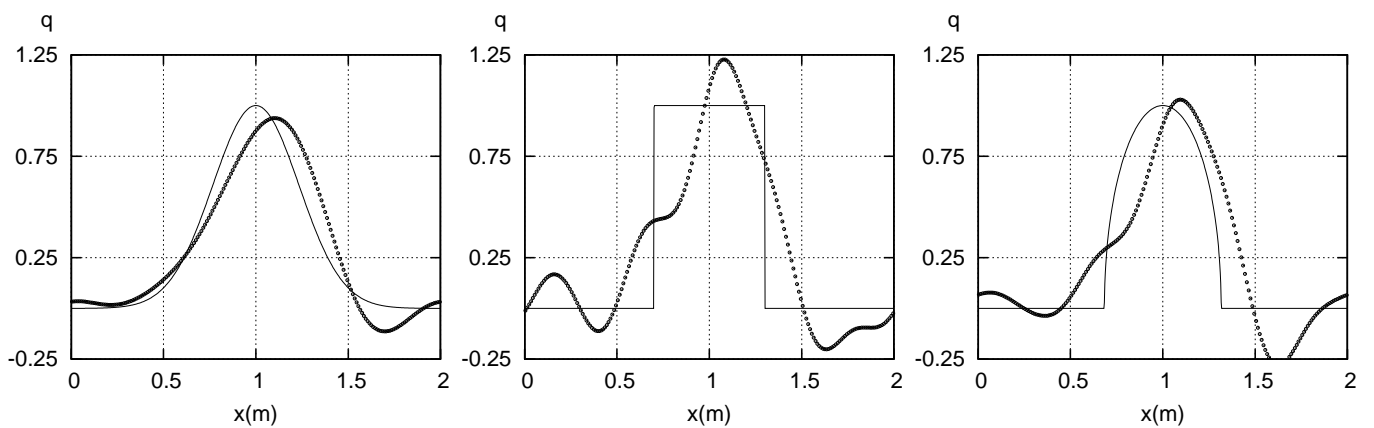

d) CFA Second Order Limited
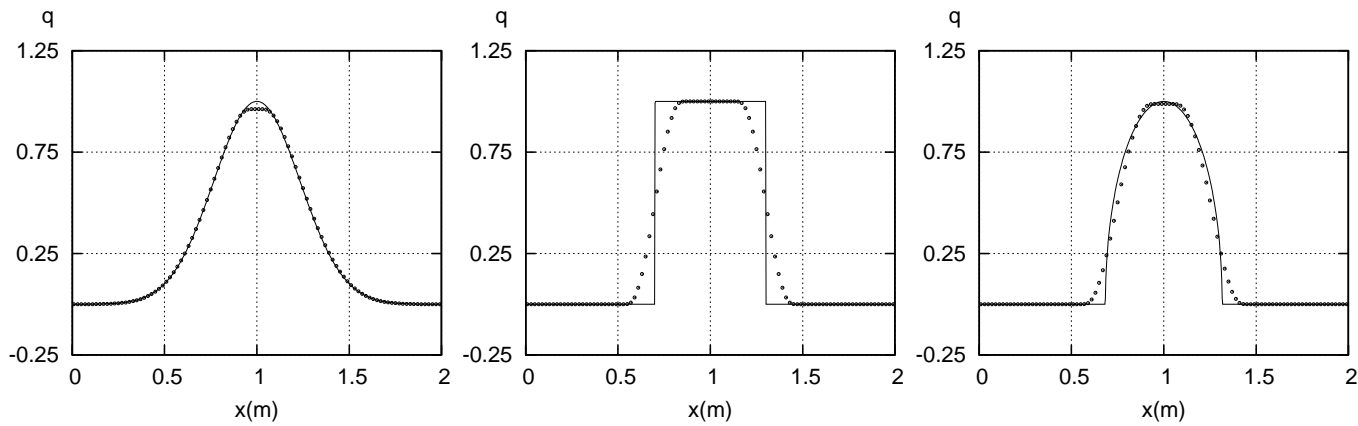

Figure 6: Results obtained assuming three different initial conditions with the methods: CFA 2nd, Lax-Wendroff, Upwind 2nd and CFA 2nd limited. 
a) CFA order $=1$

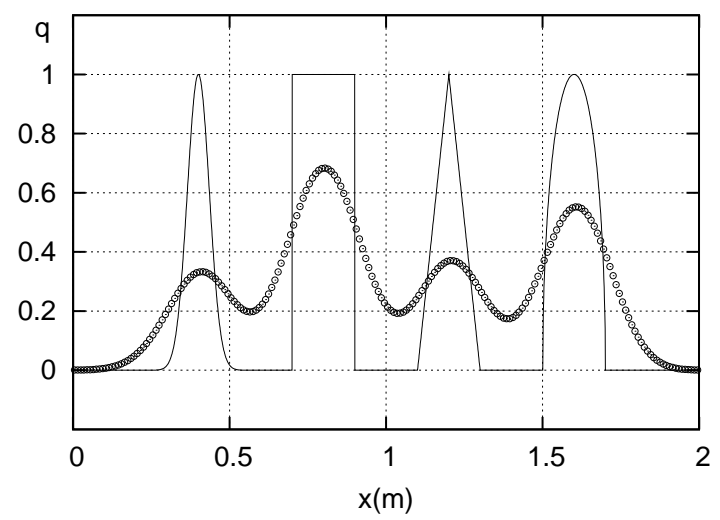

c) $\mathrm{CFA}$ order $=3$

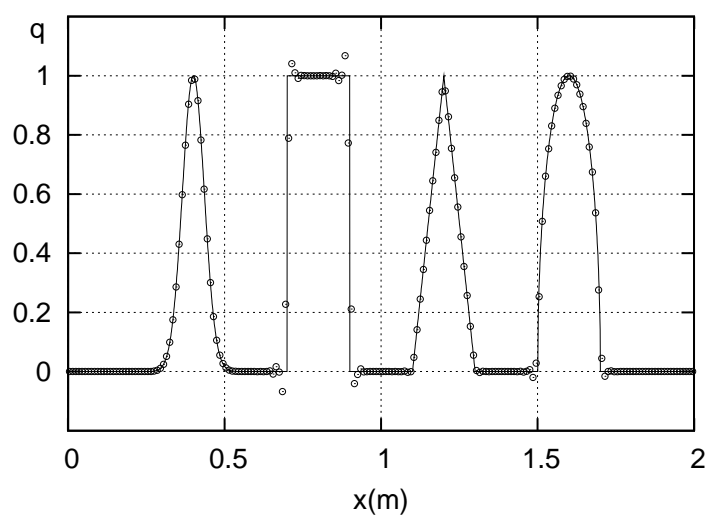

e) $\mathrm{CFA}$ order $=5$

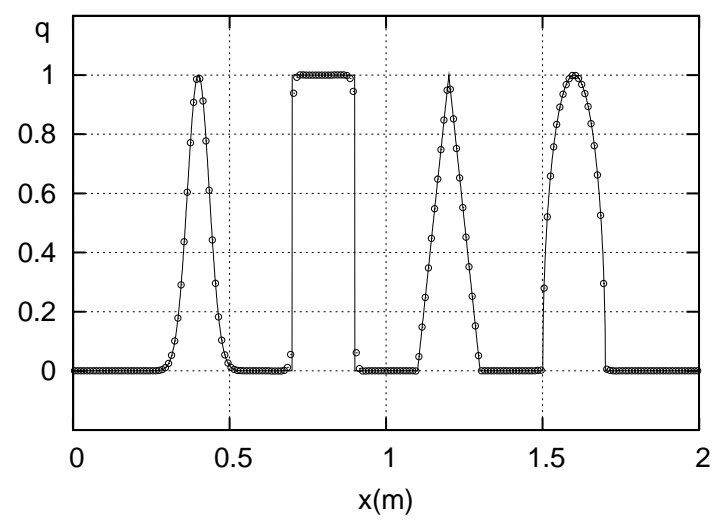

b) CFA order $=2$

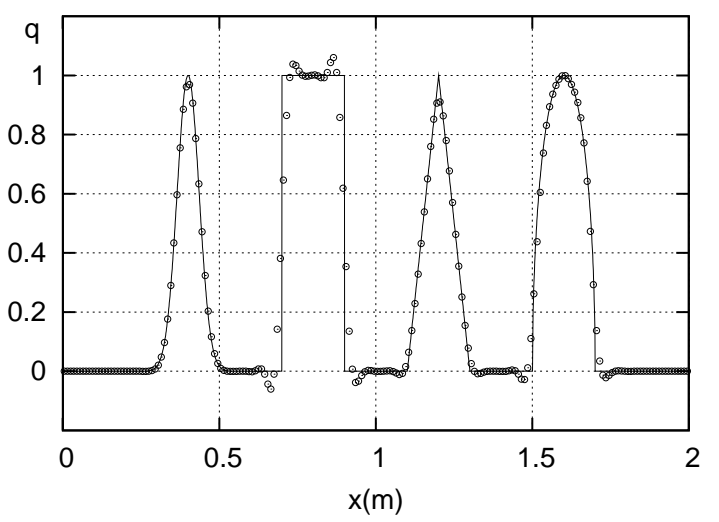

d) CFA order $=4$

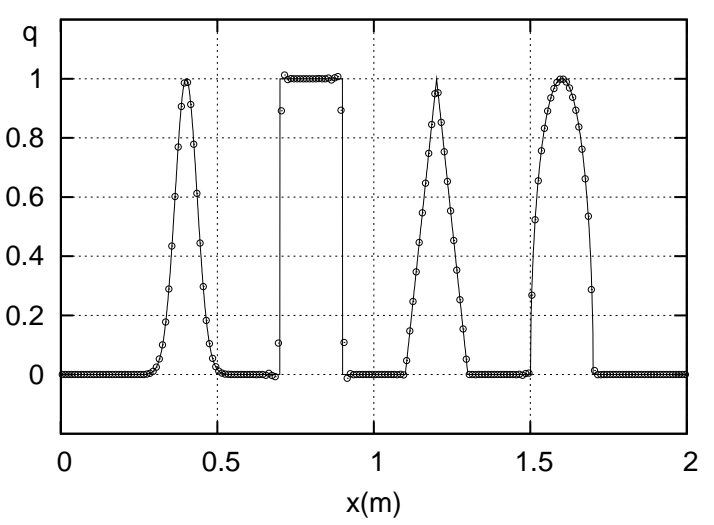

f) CFA order $=10$

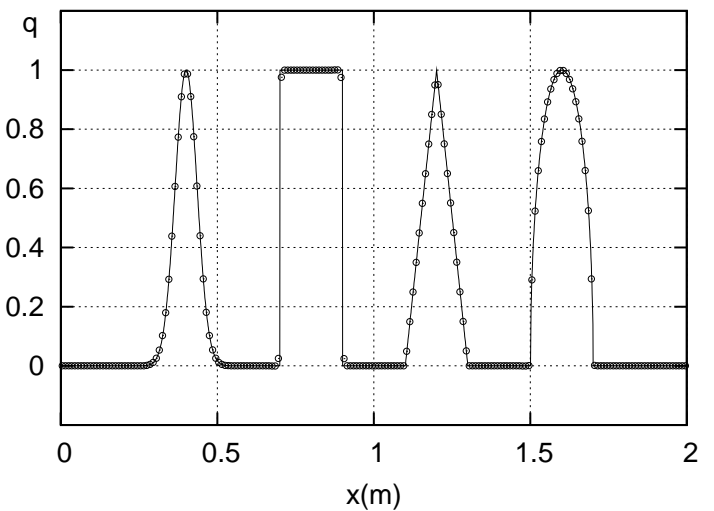

Figure 7: Average values of the numerical solution of the CFA methods of order of accuracy $1,2,3,4,5$ and 10 in numerical Test $4 .(M=200$ cells, $\mathrm{t}=20, \mathrm{CFL}=0.95)$ 
a) CFA order $=1$

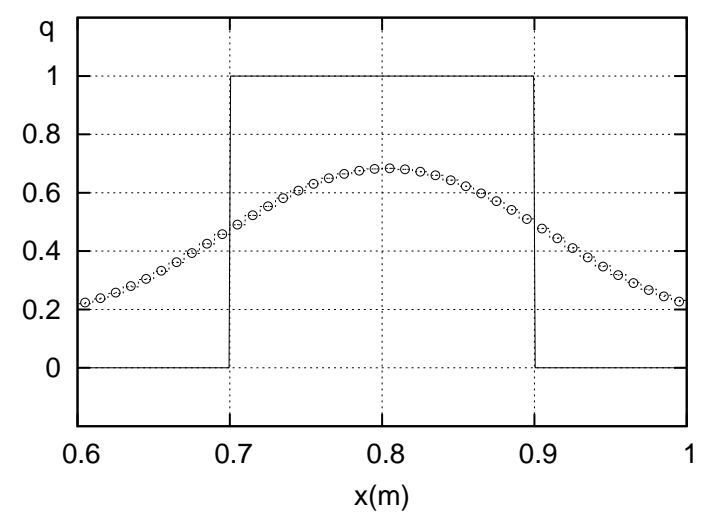

c) CFA order $=3$

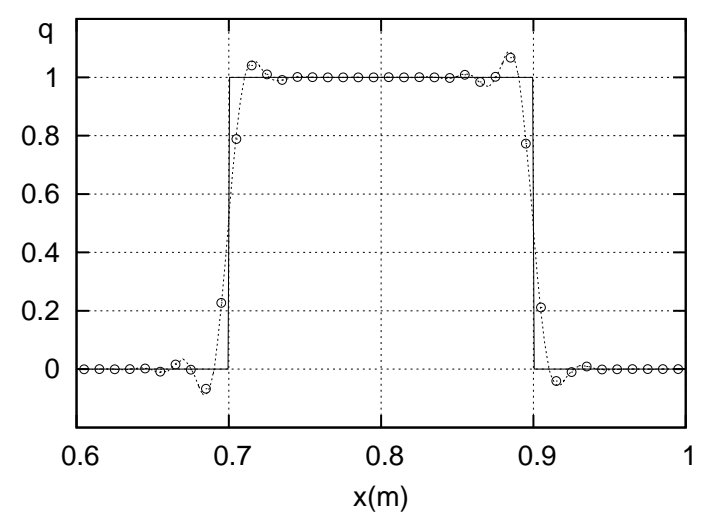

e) CFA order $=5$

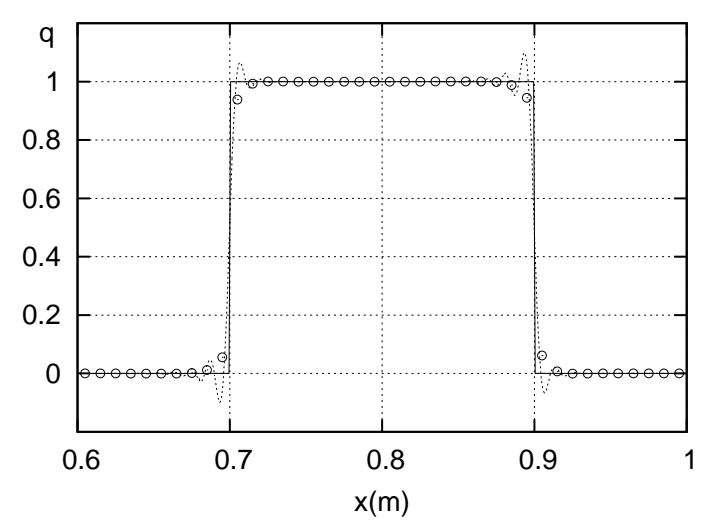

b) CFA order $=2$

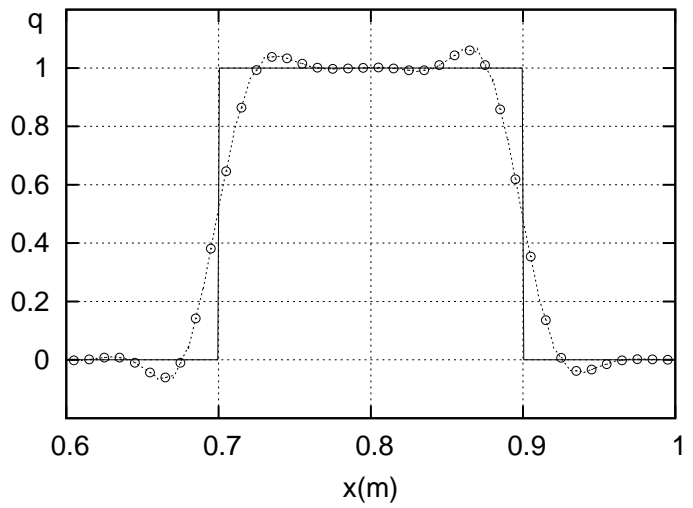

d) CFA order $=4$

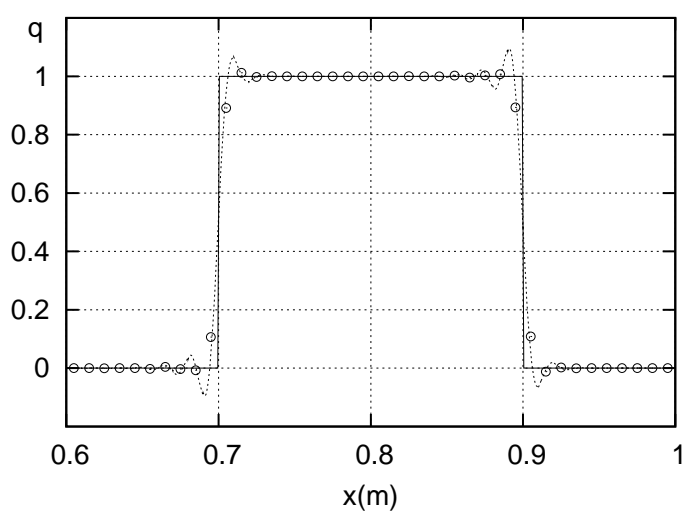

f) CFA order $=10$

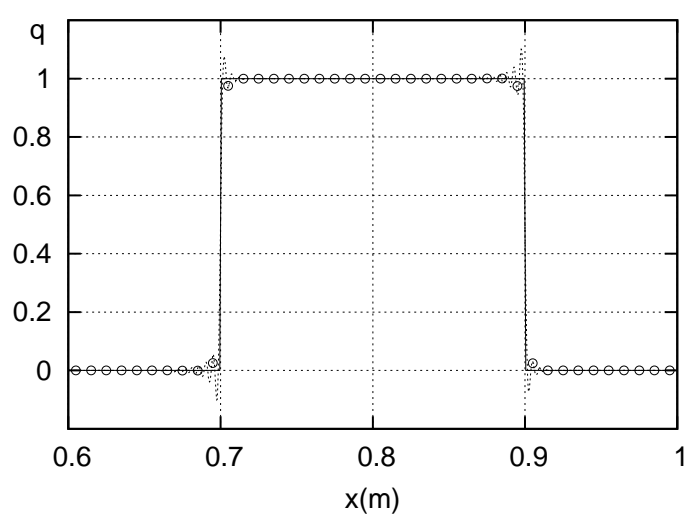

Average cell value

Subcell distribution

Figure 8: Detail of the numerical solution of the CFA methods of order of accuracy 1, 2, $3,4,5$ and 10 in numerical Test $4 .(M=200$ cells, $\mathrm{t}=20, \mathrm{CFL}=0.95)$ 
a) CFA order $=1$

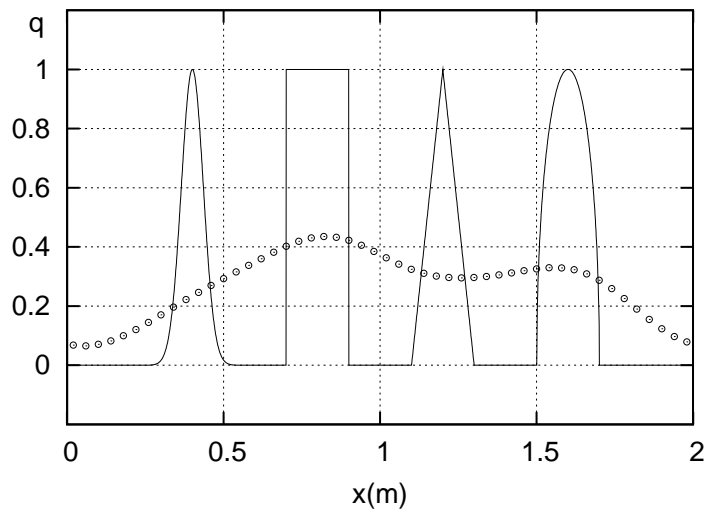

c) CFA order $=3$

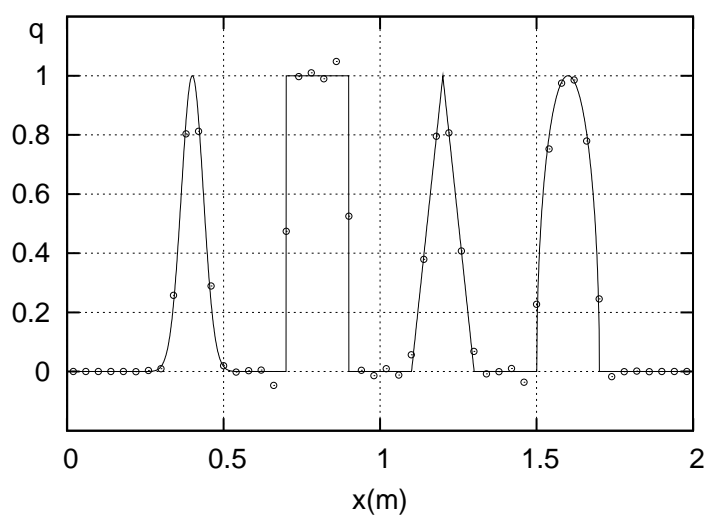

e) CFA order $=5$

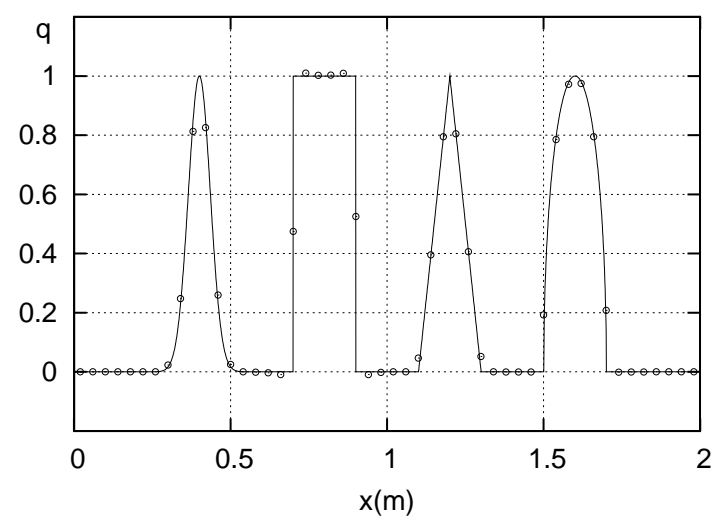

b) CFA order $=2$

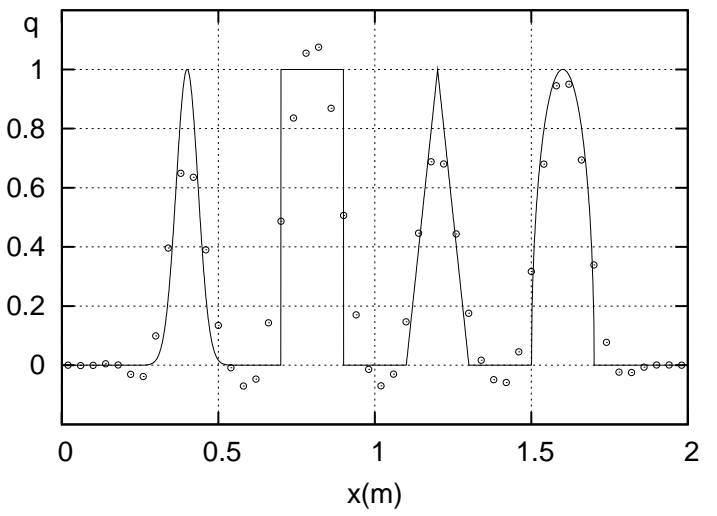

d) CFA order $=4$

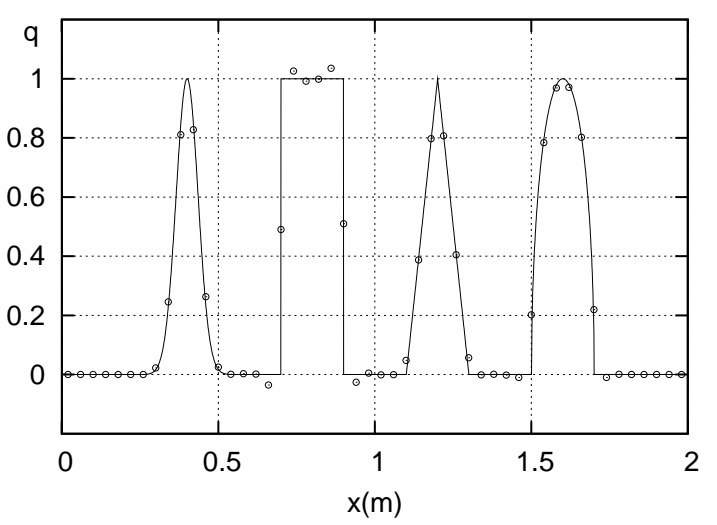

f) CFA order $=10$

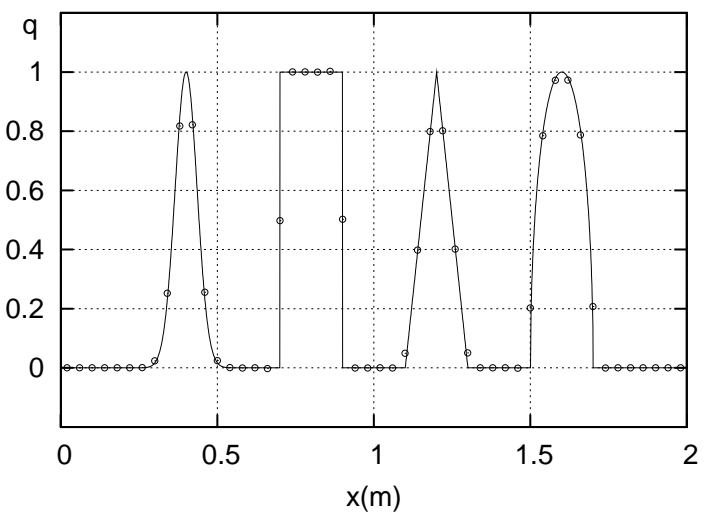

Figure 9: Average values of the numerical solution of the CFA methods of order of accuracy $1,2,3,4,5$ and 10 in Test case $4 .(M=50$ cells, $\mathrm{t}=20, \mathrm{CFL}=0.95)$ 
a) CFA order $=1$

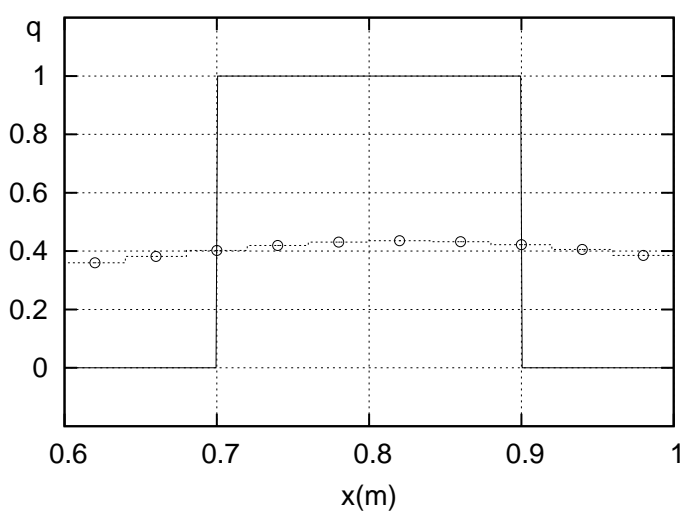

c) CFA order $=3$

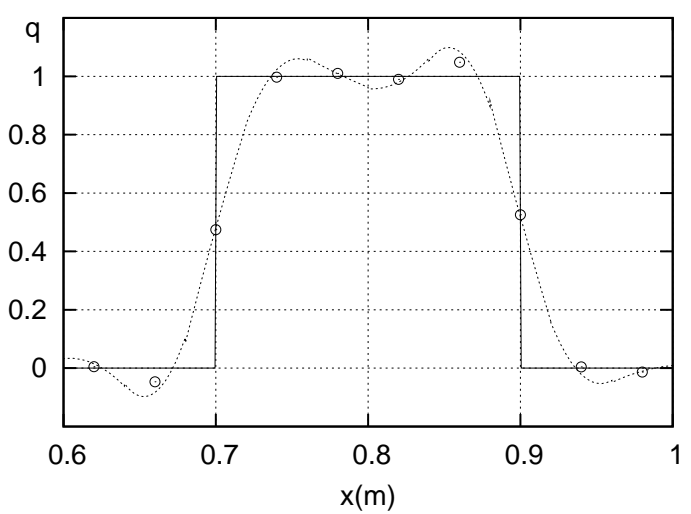

e) $\mathrm{CFA}$ order $=5$

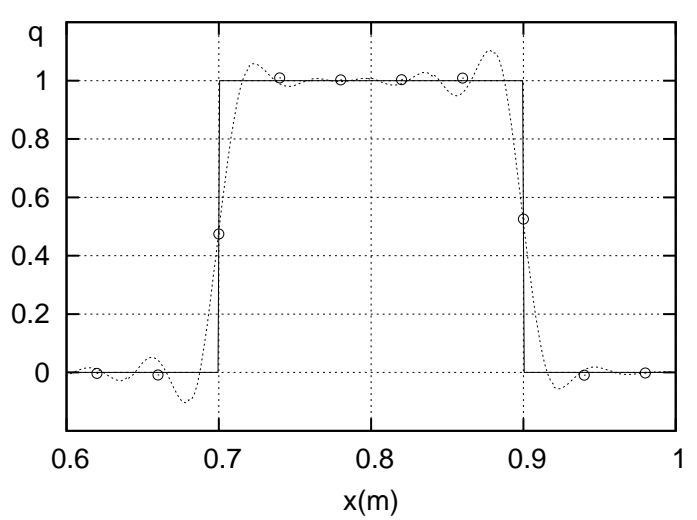

b) CFA order $=2$

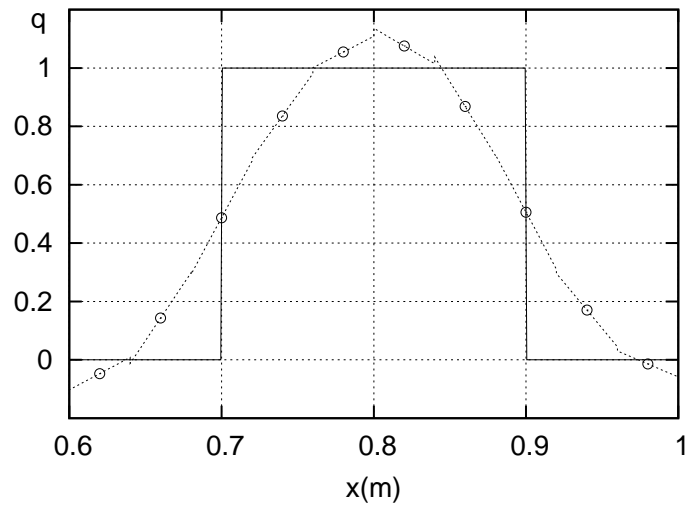

d) CFA order $=4$

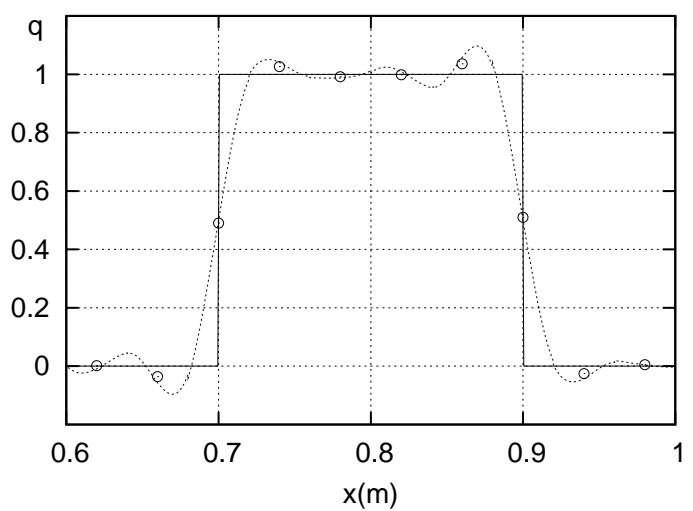

f) CFA order $=10$

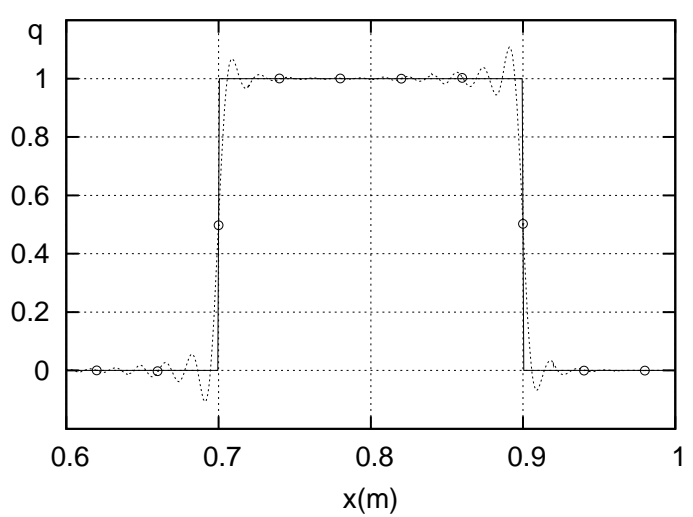

Average cell value

Subcell distribution

Figure 10: Detail of the numerical solution of the CFA methods of order of accuracy 1, $2,3,4,5$ and 10 in Test case $4 .(M=50$ cells, $\mathrm{t}=20, \mathrm{CFL}=0.95)$ 


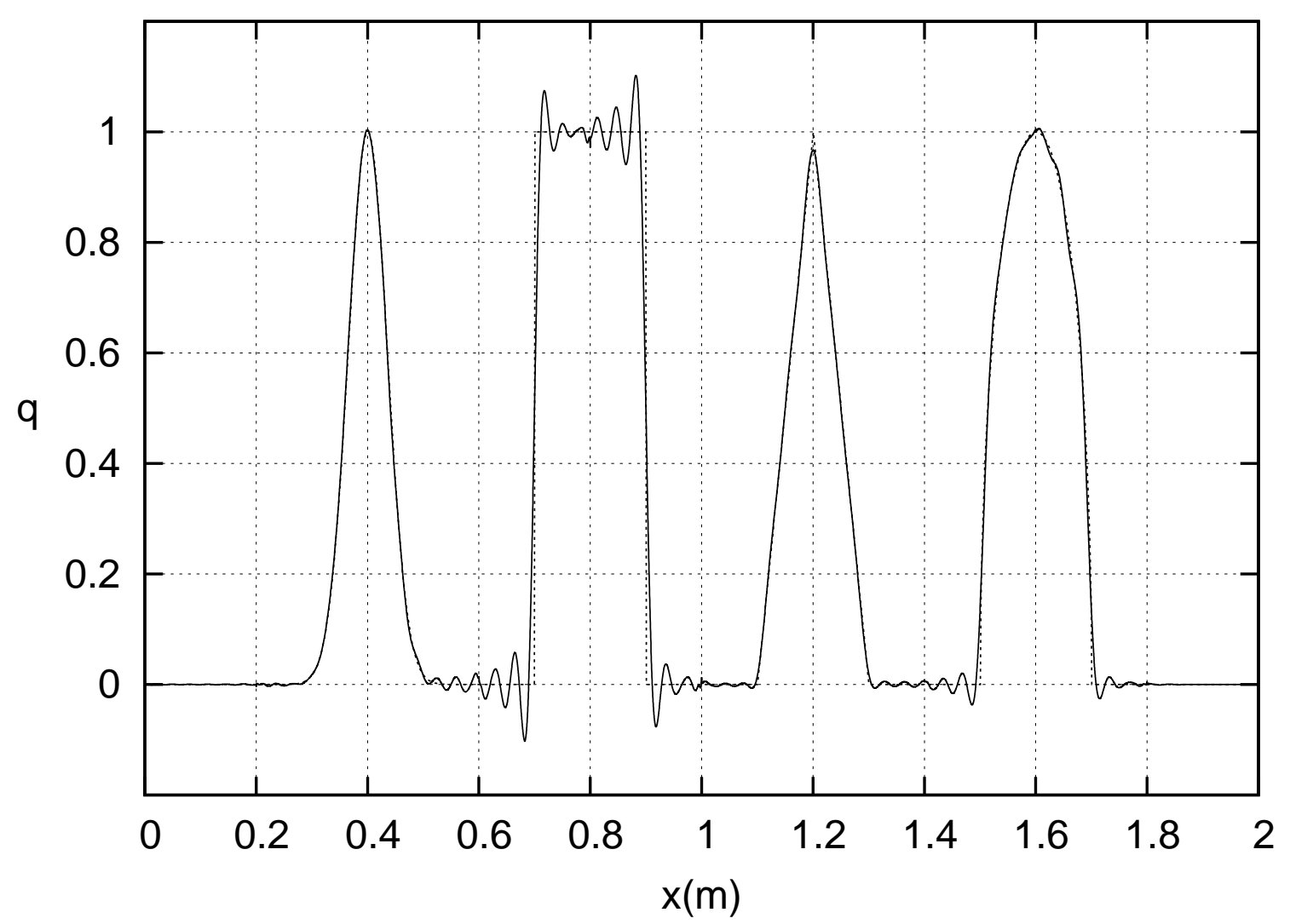

Figure 11: Numerical solution of the CFA method of order of accuracy 20 in numerical Test 4 showing the subgrid information. $(M=10$ cells, $\mathrm{t}=20, \mathrm{CFL}=0.95)$
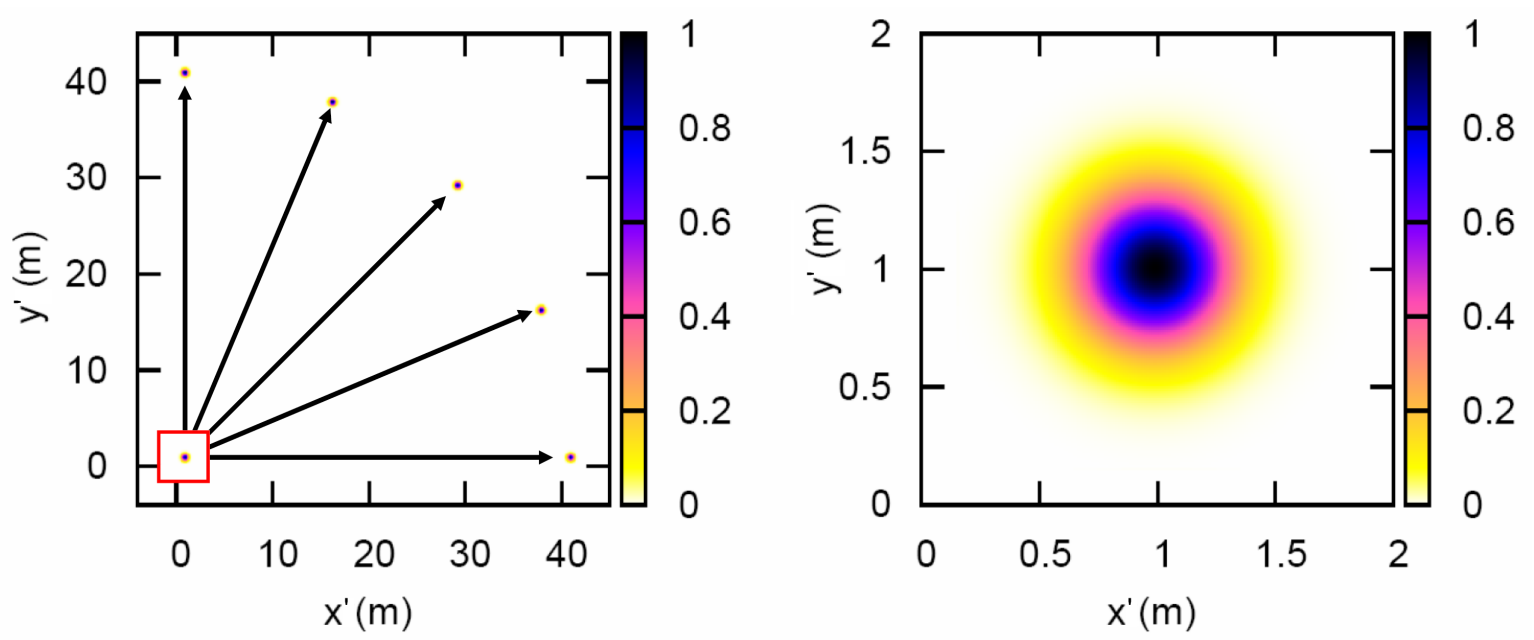

Figure 12: Description of Test case 5 representing the advection of a Gaussian initial distribution at different directions in the plane. 
a) Alfa $=0$

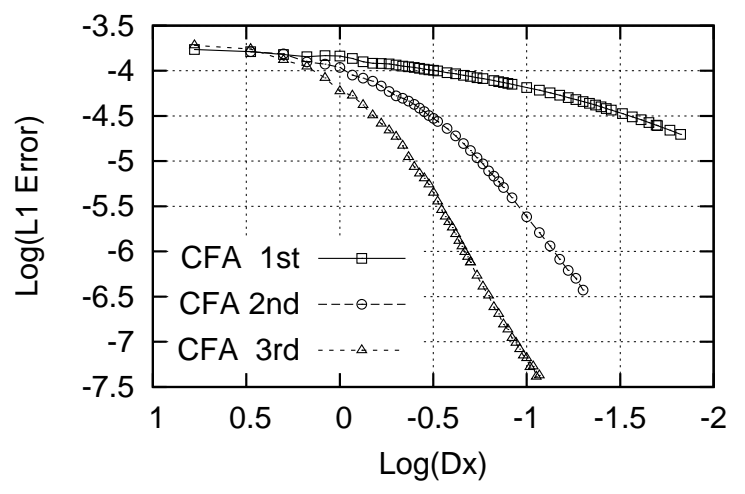

b) Alfa $=22.5$

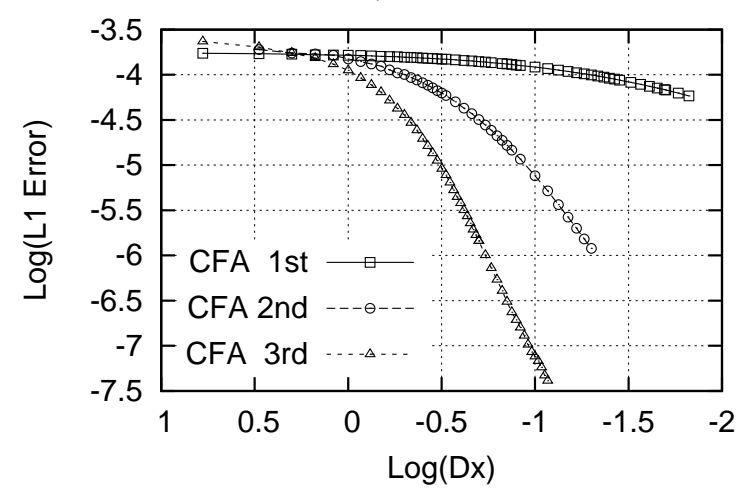

c) Alfa $=45$

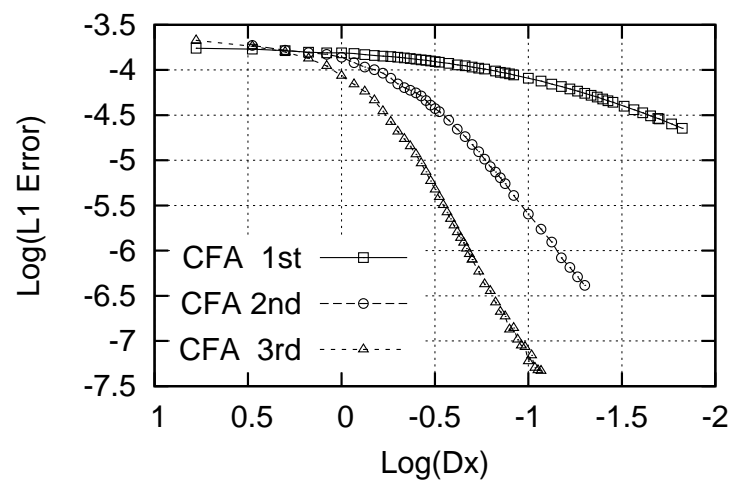

Figure 13: Error convergence of the CFA methods of order of accuracy 1, 2 and 3 in Test case 5. Results are grouped by advection direction. 
a) CFA 1st

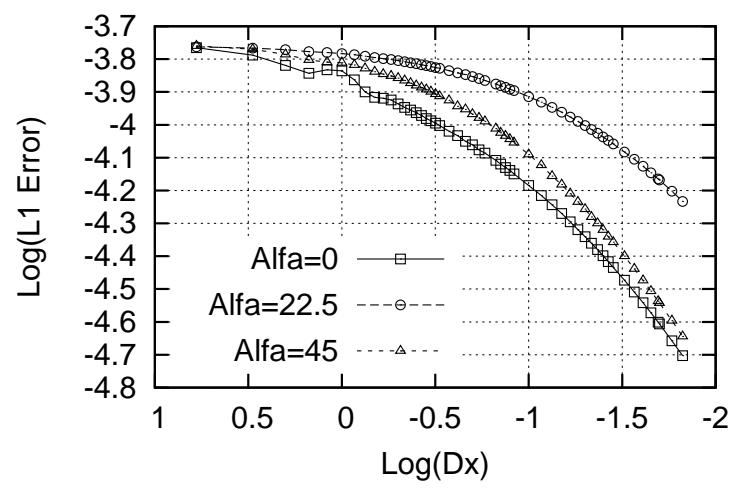

b) CFA 2nd

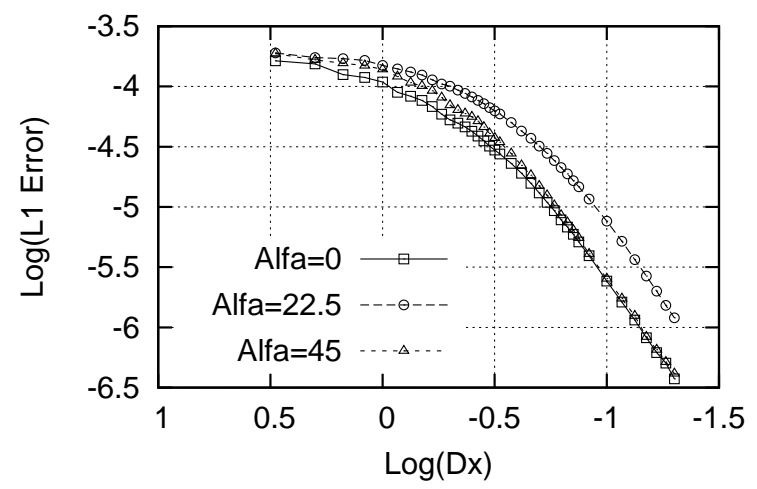

c) CFA 3rd

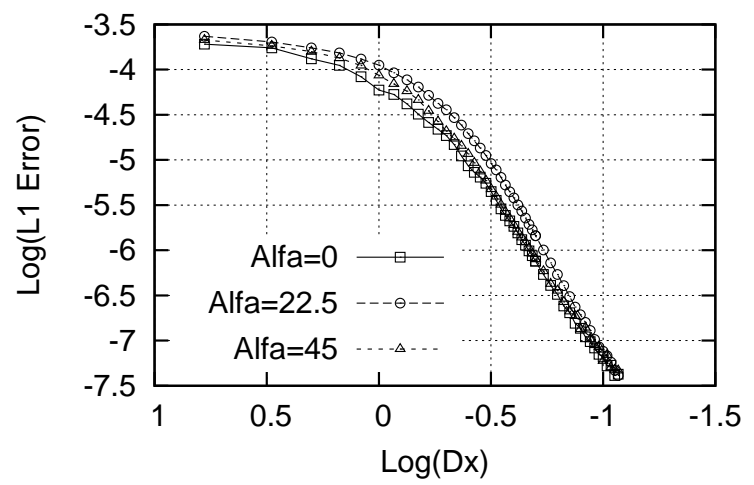

Figure 14: Error convergence of the CFA methods of order of accuracy 1, 2 and 3 in Test case 5. Results are grouped by order of accuracy.

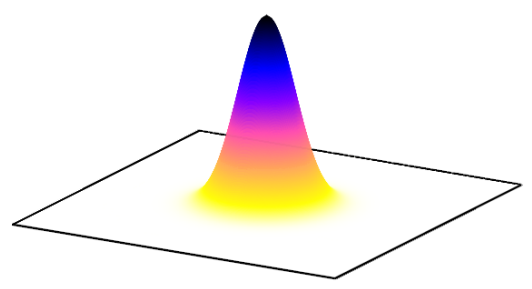

Exact Solution

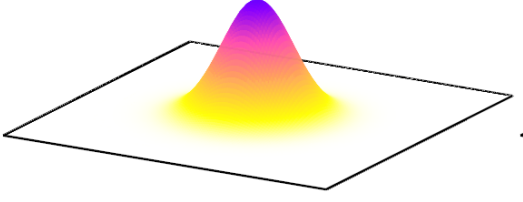

$\mathrm{L} 1=-4.5$

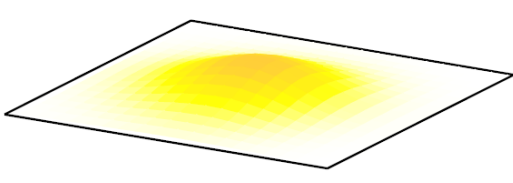

$\mathrm{L} 1=-4.0$

Figure 15: Shape of the numerical solutions corresponding to values of $\log \left(L_{1}\right)=-4.5$ and $\log \left(L_{1}\right)=-4.0$ compared to the exact solution in order to visualize the size of the $L_{1}$ error in Test case 5 . 
a) Alfa $=0$

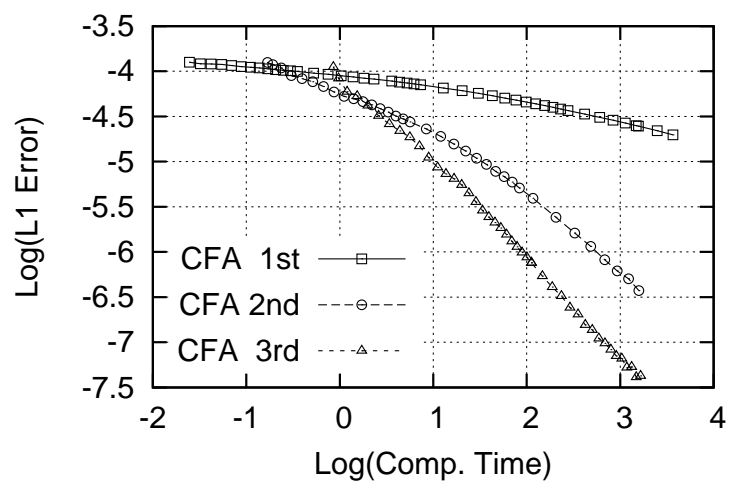

b) Alfa $=22.5$

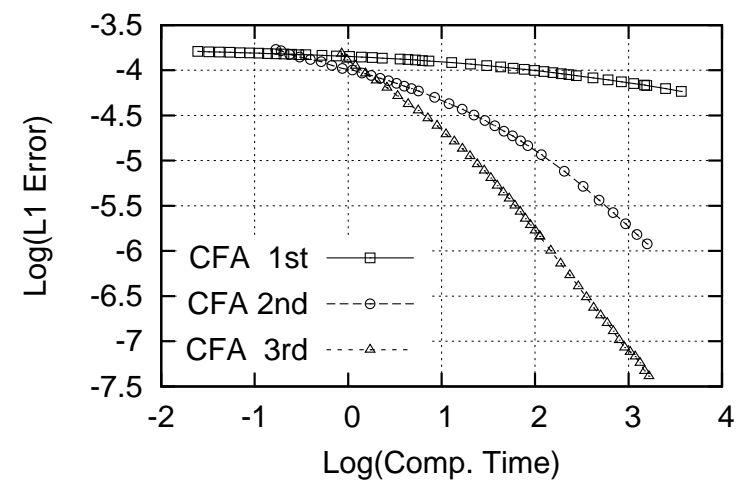

c) Alfa $=45$

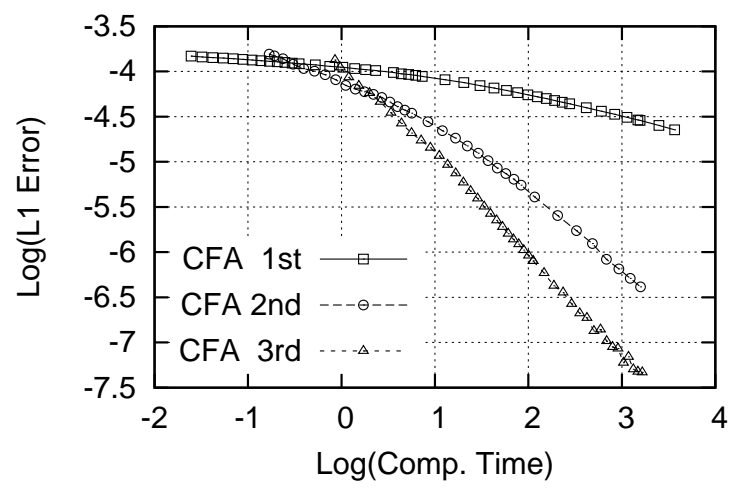

Figure 16: $L_{1}$ error norm produced by the CFA schemes of orders of accuracy 1, 2 and 3 as a function of the computational time used in numerical Test 6 . Results are grouped by advection angle. 
a) CFA 1st

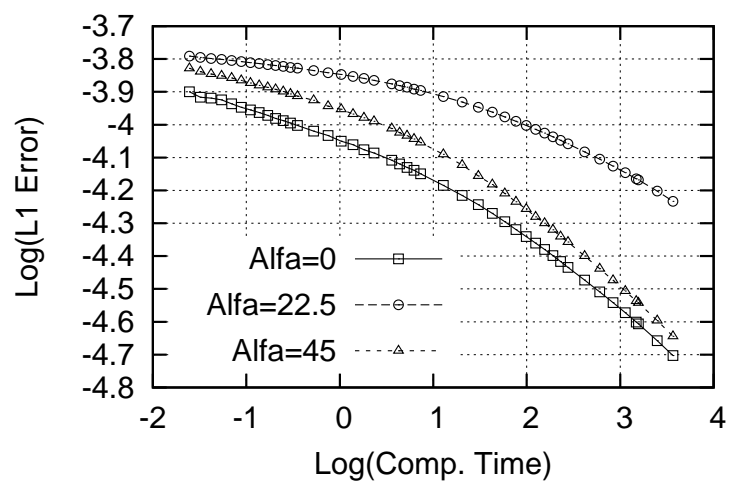

b) CFA 2nd

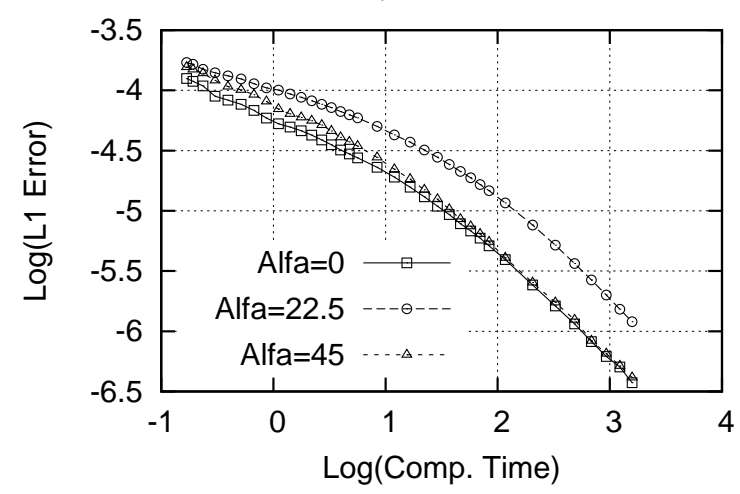

c) CFA 3rd

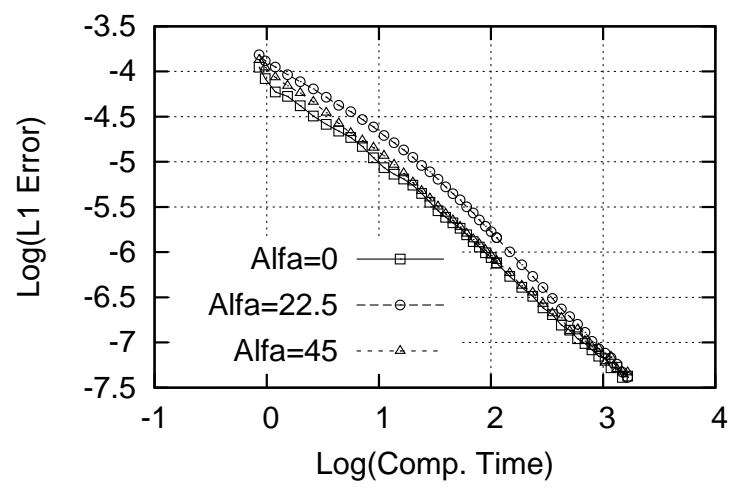

Figure 17: $L_{1}$ error norm produced by the CFA schemes of orders of accuracy 1,2 and 3 as a function of the computational time used in numerical Test 6 . Results are grouped by order of accuracy. 


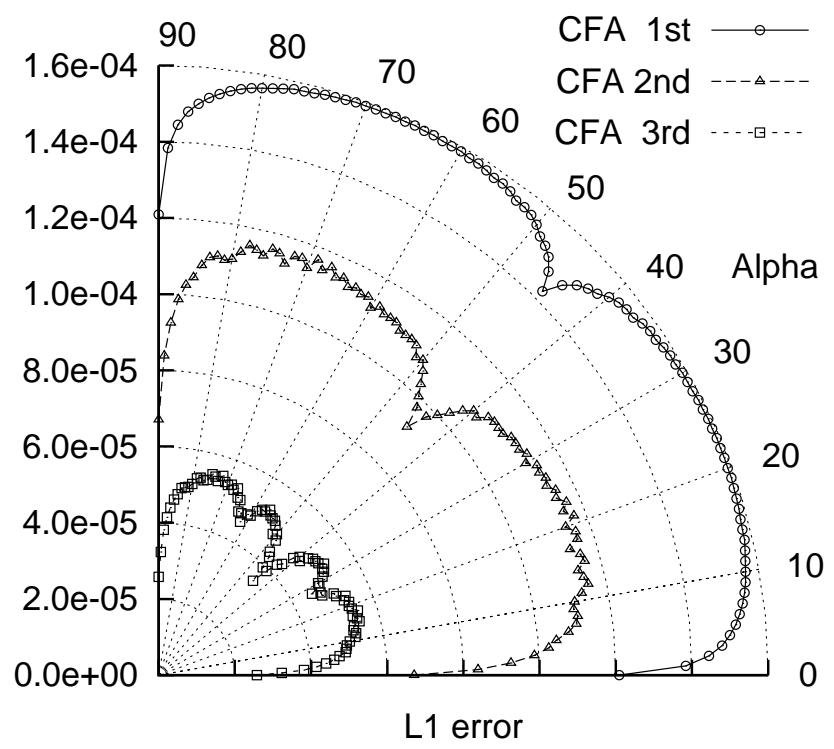

Figure 18: Angular dependence of the $L_{1}$ error produced by the CFA schemes of orders of accuracy 1, 2 and 3 in Test case 7 .

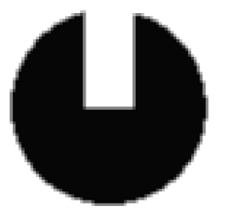

a) Exact solution b) Order=1 $150 \times 150$ cells

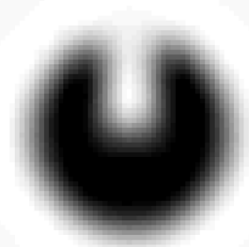

c) Order $=2$ $60 \times 60$ cells

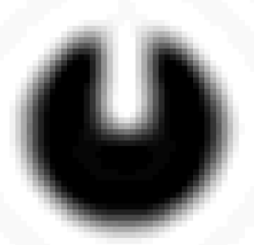

d) Order=3 $35 \times 35$ cells

Figure 19: Numerical results produced by the CFA schemes of orders of accuracy 1, 2 and 3 using different computational meshes in Test 8. The three simulations are achieved in the same computational time. 